\title{
A statistical decision model for sensory discrimination which predicts Weber's law and other sensory laws: Some results of a computer simulation
}

MICHEL TREISMAN, INSTITUTE OF EXPERIMENTAL PSYCHOLOGY, UNIVERSITY OF OXFORD, ENGLAND

A model for visual intensity threshold discrimination is described. Simplified assumptions represent the main features of the visual afferent paths. Discriminative responses are selected centrally by a statistical decision procedure, limited by the noise level. Noise arises from the irreducible physical variability of light, from spontaneous firing, and from variations in transmission in the afferent paths. These variations will tend to be positively correlated. The model was simulated on a computer: it correctly predicts the form of the Weber function (the relation between difference threshold and background intensity) and the features of spatial and temporal "summation". It also shows that the function relating the central effect of a stimulus to its physical intensity is unlikely to be logarithmic or a power function with a small exponent.

\section{The Model}

"Weber's law stands as a milestone in psychological research. It is one of the first psychological laws worthy of the name, and it may prove to be of far greater and more general significance than the differential threshold problems that provided its initial formulation. In effect, it establishes a law of psychological relativity: subjective discriminations are not bound to absolute characteristics of stimuli but to relations between them." (Berelson \& Steiner, 1964). This claim illustrates a common error among psychologists: the over-evaluation of "laws," whose discovery is taken as an end in itself for psychology. We need to substitute for this nineteenth century fallacy the appreciation that the observation of a behavioral regularity is not the conclusion to a psychological investigation, but a question inviting one; a "law" can only be said to be satisfactorily understood when we have a model of how the subject functions from which the observed behavior follows as a logical consequence. No grandiloquent generalization can substitute for this. The present paper arises from an attempt to examine whether we are yet in a position to devise such a model to account for Weber's law and some related findings (Treisman, 1964a); to examine the implications of the model devised it was simulated on a digital computer.

The model as presented is intended to apply only to the simplest detection situation: determination of the increment or absolute threshold for a flash superimposed on a background of the same area or larger. Extra assumptions will be needed to extend it to more complex situations, such as determination of the CFF, but it will be better to add these assumptions when the basic model has been evaluated. It has been applied to visual thresholds since we know more about the absorption of energy by the receptors for vision than for other senses, but in principle it is capable of being adapted to any sense.

The main assumptions on which the model rests are: (i) There is a statistical relation between the nominal intensity of a stimulus and its effect on the retinal receptors. The variation from trial to trial in the number of quanta absorbed from a light-flash of constant nominal intensity is described by a Poisson distribution. (ii) There is a statistical relation between the effect on the sense organ and the nervous message received centrally. Thus if the same number of quanta were absorbed by the receptors on more than one trial, sensory noise would nevertheless cause the number of nerve impulses arriving at the point where the threshold is determined to vary. (iii) The variation due to this sensory noise is, to some degree, positively correlated. (iv) A statistical decision procedure determines the response made.

For the most part, these assumptions are not new. The last three could be adapted to apply to any sensory system, and would then account for the occurrence of Weber's law (Treisman, 1964a). A number of lesser assumptions are included in the model to give a closer fit to the visual system, though the model should still be considered schematic, and the importance of some of these parameters and assumptions has been assessed by varying the values given to them in the computer program. The model is essentially an informationprocessing account in which the successive stages bear a rough parallel to those which physiological observations suggest may occur in the visual pathway. A brief description of the computer program will be given which will also serve as a more detailed account of the model. Some of the assumptions will then be discussed more fully.

We start by considering a single trial on which a background light-flash of intensity $I_{1}$ quanta/sec. deg. ${ }^{2}$, and area $A_{1}$ deg. $^{2}$ is exposed to the eye for $t_{1}$ sec. Steps (1) to (5) below describe how the program computes the effects of such an exposure.

(1) When a visual stimulus of given intensity is presented to the eye the number of quanta absorbed by retinal receptors will depend on the area and duration of the stimulus and the extent to which light is lost in 
the optic media or bypasses the receptors. This last is represented by $f$, the proportion of the quanta incident at the cornea which is absorbed by visual pigment. Rushton (1956) estimates that 10 per cent of the light entering the eye $\left(\lambda=505 \mathrm{~m} \mu, 20^{\circ}\right.$ parafoveal $)$ is absorbed by rhodopsin, and $f$ was always taken as 0.1 in the program, which also had, unless otherwise stated, $A_{1}=1 \operatorname{deg}_{0}{ }^{2}$ and $t_{1}=0.1 \mathrm{sec}$. Then the expected number of quanta absorbed from the flash of intensity $I_{1}$ is given by $g_{1} I_{1}$, where $g_{1}=f_{1} t_{1}$. However, noise arising in the visual system may spontaneously produce events confusable with the absorption of quanta of light by visual pigment. Following Barlow (1957) we shall treat these events as though they were always due to the spontaneous decomposition of molecules of visual pigment, and represent this retinal noise by an equivalent "dark light, " $I_{n}$, whose intensity is such that it would produce quantal absorptions at the same rate as that at which these noise events occur. Then the total expected number of quantal absorptions (using this term, from here on, to include noise events confusable with quantal absorptions, unless otherwise stated) will be given by $\mathrm{x}_{1}=\mathrm{g}_{1}\left(\mathrm{I}_{1}+\mathrm{I}_{\mathrm{n}}\right)$. The program took $\mathrm{I}_{\mathrm{n}}=1000$ quanta $/ \mathrm{sec}$. deg. 2 (Barlow, 1957).

(2) The mean number of quanta absorbed on each trial is given by $x_{1}$. But the actual number of quanta absorbed will vary from trial to trial because of the irreducible quantum variability of light. This is described by a Poisson distribution, so the program takes the actual number of decompositions of visual pigment on the given trial as $x_{2}$, where this is a variable from a Poisson distribution with mean $x_{1}$. (For $x_{1}>100$ a normal approximation to the Poisson distribution was used.)

(3) Not all the quanta absorbed may be effective at the stage at which sensory messages are set up. We assume that light adaptation acts by reducing the number of absorptions which are effective in setting up sensory messages, so that the effect of adaptation is represented by the conversion of $x_{2}$, the actual number of quantal absorptions on the given trial, to $x_{3}$, the number of absorptions effective in determining the neural message. Various assumptions might be made about this relation, since little is known about light adaptation. Those selected for inclusion in the program were:

(i) $N$ (no) Adaptation. $\quad x_{3}=x_{2}$. All absorptions are effective.

(ii) F (fractional) Adaptation. $\quad \mathrm{x}_{3}=\mathrm{hx}_{2}, 0<\mathrm{h}<1$. The number of absorptions effective is a fixed proportion of the total absorptions on a given trial. This would represent an adaptive process approximately as rapid in its effects as the direct excitatory action of the stimulus.

(iii) P (partial) Adaptation. This assumes that the momentary stimulus input can be considered to be divided into two parts, an "adaptation level" determined by the average intensity of the stimuli over a series of trials, $I_{1}$, and the deviation from this adaptation level of the given input; these two parts are differently effective at the stage at which the neural message is determined. The deviation was taken as $\mathrm{x}_{3}=\mathrm{x}_{2}-\mathrm{kx}_{1}$, with $\mathrm{k}=0.95$, and the adaptation level was then $x^{\prime \prime} 3=k x_{1}$.

(iv) C (complete) Adaptation. This is similar, except that the "adaptation level" is assumed to have no further effect. $\mathrm{x}_{3}=\mathrm{x}_{2}-\mathrm{kx}_{1}=\mathrm{x}_{2}-\mathrm{x}_{1}+\mathrm{cx_{1 }}, 0<\mathrm{c}<1$. The number of decompositions effective at the next stage is given by the excess of the actual number on the given trial over the mean number for a series of trials, plus a proportion, $c$, of this mean number. It was taken that $c=0.05$.

(v) M Adaptation (adaptation to the mean). $\quad \mathrm{x}_{3}=\mathrm{x}_{2}-\mathrm{x}_{1}$. Only the deviation from the mean level is effective at the next stage. This would imply that information about the absolute level of illumination must be conveyed by some other, presumably less sensitive, system than the one under consideration which determines intensity discrimination.

(4) We shall represent the relation between the final neural effect $(E)$ of the stimulus, and its physical intensity (I) as the overall "transducer function," $\mathrm{E}=\mathrm{f}(\mathrm{I})$. This is taken here to reflect the relation between the number of sensory messages set up, $x_{4}$, and the number of pigment decompositions effective in setting them up, $x_{3}$. For reasons which will be given below the effects of five alternative transducer functions were explored: (i) $x_{4}=\log x_{3}$; (ii) $x_{4}=x_{3}^{0.3}$; (iii) $x_{4}=x_{3}^{0.6}$; (iv) $x_{4}=x_{3} ;$ (v) $x_{4}=x_{3}^{1.4}$. The overall transducer functions resulting from these relations are represented as $E=\log I, E=I^{0.3}$, etc. In the case of partial adaptation it was assumed that the "adaptation level" is always transduced logarithmically, the functions given above applying to deviations from this level. Thus for $E=I^{1.4}, x_{4}=x_{3}^{\prime}{ }^{1.4}+\log x^{\prime \prime} 3$, for $E=\log I$, $x_{4}=\log x_{3}^{\prime}+\log x^{\prime \prime} 3$, etc.

(5) The number of sensory messages set up, $x_{4}$, has now been computed. We will suppose that each message consists of a train of nerve impulses, but any other neural coding compatible with the other assumptions of the model could be substituted for this. If the mean number of nerve impulses in a sensory message is $\mathrm{s}$, and the total number of impulses arising is summed centrally to give $\mathrm{E}$, the effect of the light-flash at the point where responses are selected, then the expected value of $\mathrm{E}$ would be $\mathrm{sx}_{4}$. But this is unlikely to be the magnitude of the central effect of the stimulus on any given trial since sensory noise will cause the actual number of nerve impulses in each sensory message to vary about $s$; it is assumed that this variation is normal, with variance $\sigma{ }_{\mathrm{s}}^{2}$. The program takes $s=1$ and treats the message size as a continuous variable with $\sigma_{\mathrm{S}}=0.1$. The "noisiness" of the sensory message is then indicated by its coefficient of variation, $v={ }_{s} / s=$ 0.1 . There is little relevant evidence on the variability 
of firing in sensory nerve fibers but it is of interest that Stein and Matthews (1965) have measured the distribution of inter-pulse intervals for cat muscle spindle afferents under constant stimulation, and found that, except at the lowest frequencies, this was normal with $\mathrm{v}$ constant, its mean value being 0.05 for primary and 0.02 for secondary afferent endings. It seems unlikely that the visual system is much noisier than the proprioceptive. As a prediction from the present model depends on $\mathrm{v}$ being small, 0.1 was taken as a conservatively large figure.

However, we are not yet in a position to determine $x_{5}$, the sum of the nerve impulses arising on the given trial, as the variance of the central effect, E, will depend not only on $\sigma \underset{S}{2}$, the variance of the sensory messages, but also on the correlations between them. As an illustration let us suppose that exactly two sensory messages arise on each of $\mathrm{N}$ trials, and that on each trial we arbitrarily label these $s_{1}$ and $s_{2}$. We could then calculate the product-moment correlation coefficient, $r_{12}$, between the magnitudes of $s_{1}$ and $s_{2}$ over the $\mathrm{N}$ trials. Sensory noise might be partly due to factors such as variation in the level of facilitation of synapses in the visual afferent pathway by the ascending reticular system or any other source, variation in oxygen supply depending on the phase of the pulsecycle, or other causes which would tend to affect different sensory messages arising at the same time in a similar way. This would make it seem likely that $\mathrm{r}_{12}$ would be positive though, of course, it could have any value between -1 and +1 . If there were three sensory messages on each of the $\mathrm{N}$ trials, then, since we label them arbitrarily, we would expect $r_{12}=r_{13}=$ $r_{23}=r$, where $r$ is the mean level of correlation between sensory messages. In this case, however, it is no longer true that $r$ can range between -1 and +1 . The possible range of values for $r$ can be shown as follows: we assume that there are $n$ sensory messages on each trial, $s_{1}, s_{2}, \ldots s_{i}, \ldots s_{n}$, with $\bar{s}_{i}=0$ and $\sigma_{i}=1$. Then

$$
\begin{aligned}
& \frac{1}{N} \sum^{N}\left(s_{1}+s_{2} \cdots s_{n}\right)^{2} \geq 0 \\
& \frac{1}{N}\left[\sum s_{1}^{2}+\sum s_{2}^{2} \cdots \sum s_{n}^{2}+\right. \\
& \left.\quad\left(\sum s_{1} s_{2}+\sum s_{1} s_{3}+\cdots \sum s_{n} s_{n-1}\right)\right] \geq 0 \\
& n+n(n-1) r \geq 0
\end{aligned}
$$

Therefore

$$
r \geq-1 /(n-1)
$$

Thus the lower limit of possible values of $r$ is given by $r=-1 /(n-1)$, where $n=x 4$. Furthermore, since we will almost always have large values of $x_{4}$, the possible negative values of $r$ will be very close to zero, so that in most cases the range can be taken as effectively 0 to +1 . To examine the effect of the degree of correla-

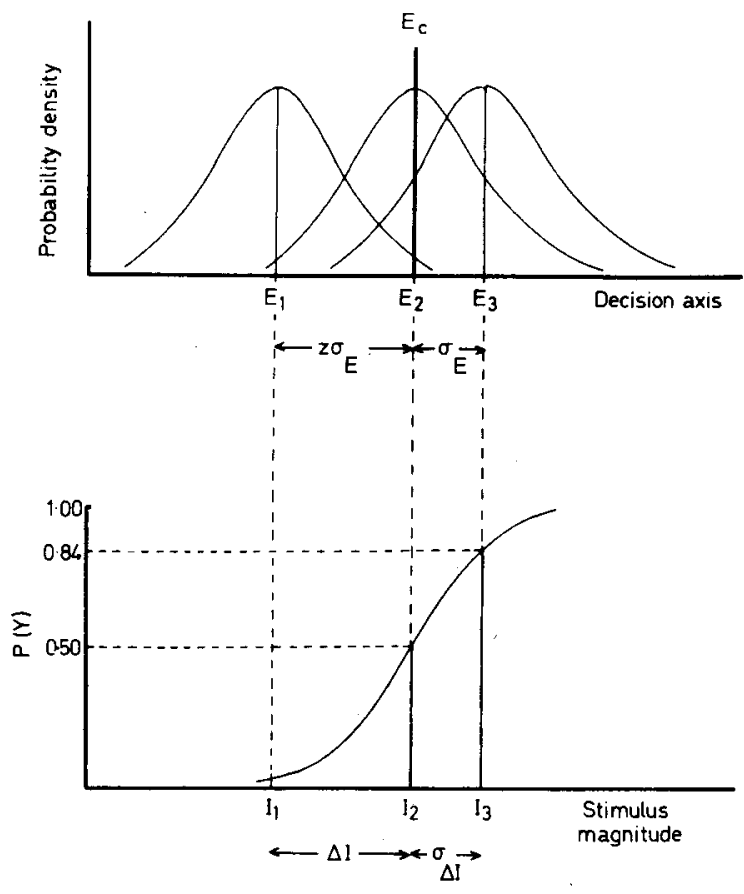

Fig. 1. The relation between the physical stimulus intensity, $I$, and its central effect, $E$, underlying measurement of the absolute or incremental threshold by the method of constant stimuli. The psychometric function relates $P(Y)$, the probability of the response 'Yes', to the physical magnitude of the stimuli presented. The three distributions on the central decision axis, $E$, correspond to three stimuli, $I_{1}$, the background stimulus $\left(I_{1}=0\right.$ when the absolute threshold is measured), $I_{2}$, the background + increment whose mean central effect coincides with the criterion, $E_{c}$, and $I_{3}$, a stimulus whose mean central effect, $E_{3}$, is exactly one standard deviation above $E_{c}$, and for which $P(Y)$ is, therefore, 84 per cent. $I_{2}-I_{1}=\Delta I$.

tion between sensory messages, $r$ was taken as $0,0.2$, $0.5,0.6$, or 1.0 , and $x_{5}$, the total number of nerve impulses arriving centrally on the given trial, was taken as a variable from a normal distribution with mean $x_{4}$ and variance determined by $\sigma \frac{2}{2}$ and $r$.

We have now given an account of how a single presentation of the stimulus might produce a central effect, $E$, which the computer calculated as $x_{5}$. However, we are interested in making predictions about the difference threshold, $\Delta \mathrm{I}$, or absolute threshold, $\mathrm{I}_{\mathrm{O}}$. Before describing how the program determines these measures, we shall briefly elaborate the assumption that threshold responses are determined by a statistical decision procedure.

Figure 1 illustrates assumptions about the determination of absolute or difference thresholds which have been put forward by a number of authors, but which are perhaps best known and have been most energetically developed as the basis of modern signal detectability theory; as this theory, and the considerable evidence for it, are well described elsewhere, only a brief account of the determination of incremental and absolute thresholds will be given here (Cattell, 1893; Solomons, 1900; Thurstone, 1927; Rose, 1948; Tanner \& Swets, 1954; Gregory, 1956; Barlow, 1956; Swets, Tanner, \& 
Birdsall, 1961; Swets, 1964; Treisman, 1964a, 1964b, 1965; Treisman \& Watts, 1966). We start with the assumption that there is a statistical relation between the central effect of a stimulus at the locus where the threshold response is determined-the "decision axis," $\mathrm{E}$-and its physical magnitude. Let us suppose that a stimulus, of intensity $I$, is repeatedly presented to a subject. Then, because of physical and sensory noise, the stimulus will not have identically the same central effect each time it is presented; instead its different central effects can be described by a frequency distribution, which is assumed to be approximately normal. Figure 1 shows three such distributions, centered on $E_{1}, E_{2}$ and $E_{3}$, the expected central effects of three stimuli, $I_{1}, I_{2}$ and $I_{3}$; to a first approximation it is taken that their variances, $\sigma \frac{2}{\mathrm{E}}$, are equal.

In the determination of an incremental difference threshold by the method of constant stimuli, $I_{1}$ would represent the background or steady stimulation. On some trials $I_{1}$ is presented, and on other trials one of a range of stimuli greater than $I_{1}$ is given. Each time the subject must respond "Yes" or "No" to indicate whether or not he considers that an increment has been added to $I_{1}$. Since the central distributions overlap, the value of $E_{i}$ occurring on trial (i) is not an unambiguous indicator of which stimulus was in fact presented on that trial. Statistical decision theory shows that in such a case the optimal procedure for the subject is to select a value on the decision axis, $E_{c}$, as a criterion, and to make each decision by reference to it: if the central effect on a given trial exceeds $\mathrm{E}_{\mathrm{C}}$ he should respond "Yes," if it does not he selects the response "No." Then the probability that the subject will respond "Yes," $P(Y)$, on trials when a given stimulus is presented, is given by the proportion of the corresponding central distribution which lies to the right of $\mathbf{E}_{\mathrm{C}}$. Figure 1 shows the psychometric function which results when $P(Y)$ is plotted against the stimulus values. For $I_{1}$, the value of $P(Y)$ is the "false positive rate," the proportion of the distribution centered on $E_{1}$ which exceeds $E_{C} . I_{2}$ happens to be the stimulus whose mean central effect, $E_{2}$, coincides with the criterion, $\mathrm{E}_{\mathrm{c}}$; it follows that for this stimulus $\mathrm{P}(\mathrm{Y})=0.5$. In general, as larger values of I are taken $P(Y)$ increases, giving a psychometric function which is a normal ogive. $P(Y)=0.5$ is usually taken as the experimental criterion defining the incremental threshold; it follows that $\Delta \mathrm{I}$, the threshold increment, is given by $\Delta I=I_{2}-I_{1}$. When $I_{1}=0$ the mean value of $E_{1}$ will be due to the retinal noise, $I_{n}$, and $I_{2}$ will now be taken as the absolute threshold for light, $I_{0}$. The distribution corresponding to $I_{1}$ is sometimes called the "noise" (N) distribution, the others being "'signal + noise"' (SN) distributions.

There are a number of ways in which a subject might settle upon a value for $E_{c}$ (Birdsall, 1955), but the basis proposed by Neyman and Pearson (1933) is the most plausible for the conventional psychophysical methods (Treisman, 1964a, 1964b, 1965). This requires that the false positive rate must not exceed an acceptable limiting value, and it is satisfied if $\mathrm{E}_{\mathrm{C}}=\mathrm{E}_{1}+\mathrm{z} \sigma_{\mathrm{E}}$, where $z$ is a constant such that $P\left(Y \mid I_{1}\right)$ is equal to the acceptable false positive rate. Since the central distribution is taken to be normal, $\mathrm{z}$ is the standardized normal deviate which cuts off a tail whose area is equal to the acceptable limiting false positive rate.

Returning to the computer program, we can see that $\mathrm{x}_{5}$, as determined above, corresponds to a value for $\mathrm{E}$ produced by a single presentation of $I_{1}$, the background stimulus. In order to determine the threshold intensity increment for $I_{1}$ we need to determine $E_{C}=E_{2}$ and then find the corresponding value of $\mathrm{I}_{2}$. Then we can obtain $\Delta \mathrm{I}=\mathrm{I}_{2}-\mathrm{I}_{1}$.

(6) Steps (1) to (5) above were repeated 1000 times, the resulting 1000 values of $x_{5}$ representing the $E_{1}$ distribution. The acceptable limiting false positive rate was taken as 2 per cent; a value, $x_{6}$, was found which was exceeded by 20 of the calculated values of $x_{5}$. This was taken as an estimate of $\mathrm{E}_{\mathrm{c}}$, the corresponding value of $\mathrm{I}_{2}$ was determined and $\Lambda \mathrm{I}$ was derived from it.

\section{Further discussion of the model}

We have now described the model and the computer program embodying it. This was used to examine the form of the Weber function (i.e., the relation between $\Delta \mathrm{I}$ and $\mathrm{I})$ and also the implications of various assumptions about light adaptation, the form of the transducer function, and the level of correlation. Before considering the results some points deserve further discussion.

(1) The transducer function: (i) The logarithmic function, $E=\log I$, which corresponds to $x_{4}=\log x_{3}$ in the program, has wide support, partly for the reasons advanced by Fechner (1860), and partly because it is believed that it provides a basis for explaining Weber's law, since if $\Delta I \mid I$ is constant, all values of $\Delta I$ will correspond to the same fixed difference after the logarithmic transformation (Gregory, 1956; Le Grand, 1957; Rushton, 1961). However, Fechner's arguments were based on his particular model for the threshold; if that is rejected his scaling procedure and his conclusions must fall away. A scaling procedure can be derived from the statistical decision model of the threshold presented above; when this is used it does not give a logarithmic function but suggests, for vision, that the transducer function is a power function with an exponent in the neighborhood of one (Treisman, 1965).

Evidence from a different source appears to be provided by the finding that category scaling and related procedures lead to a logarithmic function, and it has also been argued that the results of "direct" sensory scaling experiments, although usually taken as evidence for a power function psychophysical law, can be as well, or better, interpreted as supporting a logarithmic law (Stevens, 1957, 1960; Treisman, 1964c, 1964d). But it has also been proposed that the dimension scaled by "direct" and category scaling procedures is not the 
same as the discriminal dimension serving as decision axis in the determination of discrimination responses, but is a more central metric dimension storing information about the magnitudes of stimulus inputs; this metric dimension might be related to stimulus intensity by a logarithmic psychophysical law without this carrying any implication about the form of the transducer function (Treisman, 1965). Thus the psychophysical evidence for a logarithmic transducer function is weak; nevertheless, it seemed of interest to include it.

(ii) "Direct" scaling methods lead to a psychophysical law which, for brightness, is held to be a power function with an exponent of about 0.3 (Stevens, 1957, 1960). Although these data could be equally well accommodated by a logarithmic function with a slope constant of 0.3 (Treisman, 1964c, 1964d), and should be interpreted as relating to the metric dimension, and not the discriminal dimension with which we are now concerned (Treisman, 1965), it seemed of interest to include $\mathrm{x}_{4}=\mathrm{x}_{3}^{0.3}$.

(iii) Though implicit in much work that has been done, the linear law $\left(x_{4}=x_{3}\right)$ and similar functions do not appear to have been systematically evaluated. They may have seemed implausible because the range of light intensities over which the eye functions is so large when compared with the range of useful firing rates of a nerve fiber, but this is not an insuperable objection: (a) rods and cones function mainly over different ranges of intensity; (b) light adaptation may greatly reduce the firing rates required (partly for this reason a number of adaptation functions were included in the program); (c) we are concerned with the transducer function as it applies to the experimental measurement of visual thresholds: in these situations the eye is usually adapted to a fixed background intensity, and the decrements or increments to this intensity are small. Thus the linear law need apply to only a small range of variation about an adapting intensity, while another law could apply to the mean effect of the background intensity itself. This possibility was included in the program as $\mathrm{P}$ adaptation, the effect of the "adaptation level" being assumed to be logarithmic, whatever the transducer function applying to deviations from this level might be.

Evidence in favor of a linear or near-linear law includes: (a) A scaling procedure based on the statistical decision threshold model has been applied to data on brightness discrimination; the results obtained suggested that the transducer function is a power function with an exponent varying above and below unity. For individual subjects the exponent may be somewhat greater or less than one (Treisman, 1965). (b) Complete temporal summation is found for threshold stimuli whose duration is less than a limiting value which we will call $\tau$ (Bloch's law), and complete spatial summation (Riccò's law) is found below a limiting area, $\propto$; below these limits the threshold intensity is inversely proportional to the duration or area of the stimulus: $I_{0} t=k$ or $I_{0} A=k$, where $k$ is a constant. These laws apply for both absolute and difference thresholds (Barlow, 1958); they imply that the distribution of the stimulus energy over time and area has no effect on its discriminability, which could hold only if a linear function of energy is transmitted to the stage at which summation occurs. (c) If a succession of flashes is given at a rate above the critical flicker fusion frequency, then their apparent brightness matches that of a constantluminance equal to the mean luminance of the light-dark cycle over one period (the TalbotPlateau law). This appears to be the equivalent of Bloch's law for brightness discrimination (Le Grand, 1957; Piéron, 1965). This law holds very exactly; since, above the CFF, variation in the distribution of light over time does not affect its apparent brightness, a linear function is again implied. (d) Threshold and brightness summation might be attributed to a linear relation at the retina, with non-linearity beyond that level. However, Levelt (1965a, 1965b) has studied binocular brightness averaging and has found that for brightnesses sufficient to allow contour formation the combination of the information from the two eyes "can be simply described as an averaging of energies." For a constant comparison luminance, $\mathrm{C}$, the equibrightness curve is described by $w_{1} E_{1}+w_{r} E_{r}=C$, where $w_{1}$ and $w_{r}$ are constants adding to one, and $E_{1}$ and $E_{r}$ are the luminances of the left and right test fields. Thus at the central locus where the information from the two eyes is combined, the inputs summated are linear functions of the light energy entering the eye.

We may also note that for light signals a linear detector is the most efficient (Jones, 1959) and Rushton (1961) has proposed that neural transmission, from synapse to synapse, is linear. All these lines of evidence support the possibility of a linear transducer function $\left(x_{4}=x_{3}\right)$. It is also possible that, either as a result of individual variability, or as a result of some feature of the experimental situation, the transducer function may not be quite linear, power functions with exponents somewhat above or below one occurring, and for this reason $\mathrm{x}_{4}=\mathrm{x}_{3}^{0.6}$ and $\mathrm{x}_{4}=\mathrm{x}_{3}^{1} .4$ were included.

(2) Where are threshold responses determined? Before discussing some physiological evidence on the form of the transducer function, it is of interest to consider at what level the threshold response might be selected.

It is generally accepted that the threshold is not determined at the level of the rods (Pirenne, 1956; Rushton, 1963, 1965a). Hecht, Shlaer, and Pirenne (1942) proposed that at the absolute threshold a flash of light was seen when the number of quanta absorbed from it by rods lying within a sufficiently small area exceeded a minimum number, such as six. A simple interpretation of this might be that each rod absorbing a quantum gives rise to an impulse, and these impulses converge on a single ganglion cell which will only fire when signals arrive in sufficient number, numbers of 
impulses less than this minimum having no further effect. The subject would then respond "Yes" only if the ganglion cell fired. This account is not consistent with the threshold model given earlier, since the criterion is fixed, and input values falling below the criterion are completely lost. An experiment by Barlow (1956) shows that this model can be rejected. If, in Fig. 1, the criterion, $E_{c}$, is moved to the left there will be a rise in false positive rate (which may be small or large, depending on the initial position of $E_{c}$ ) and a fall in threshold, since the psychometric function will shift to the left, but its slope should remain the same. This describes an expectation of the statistical decision model. But if the threshold response depends on whether or not a ganglion cell fires, then values of $E$ falling below $\mathrm{E}_{\mathrm{c}}$ are not recorded so that the subject can only increase $P(Y)$ by guessing. If he guesses at a given rate, G, this will produce a corresponding false positive rate, and $P(Y)$ for eách stimulus will increase. This increase will be greater for the weaker stimuli, so that the psychometric function becomes flatter since the new probability of positive response, $\mathrm{P}^{\prime}(\mathrm{Y})$, is given by $\mathrm{P}^{\prime}(\mathrm{Y})=\mathrm{P}(\mathrm{Y})+\mathrm{G}[1-\mathrm{P}(\mathrm{Y})]$. Barlow measured the absolute threshold with the subject applying a strict and a lax criterion; with the latter there was a considerable fall in threshold with little change in the slope of the psychometric function, and the false positive rate increased only from 0 to 1 per cent. Thus it appears that the absolute threshold is not a fixed quantity determined by the firing threshold of the ganglion cell. Information provided by quantal absorptions below the critical number is available to determine positive responses if the subject lowers his criterion.

However, this does not altogether exclude the ganglion cell as a possible site of threshold determination, if we allow that its firing threshold could be variable and subject to centrifugal control. Rushton (1963, 1965a) places the site of threshold determination at this level: he relates the difference threshold to the level of firing of rods in the receptive field converging on a single ganglion cell caused by the background stimulation. He calls this the "summation pool" and proposes that threshold is reached when the input to the pool arising from the stimulus increment is sufficiently large as compared with the rate at which signals resulting from the background excitation arrive.

However, there is evidence that the outputs of different ganglion cells may contribute to determining the threshold response. There may be interference or they may combine to determine a positive response when individually they would have been insufficient: (a) "Partial areal summation" refers to a fall in threshold as the stimulus area is increased beyond the limits of Ricco's law, thus bringing in new receptive fields. (b) Pirenne (1962a) has shown that the threshold for a test flash $0.1^{\circ}$ in diameter, falling in the center of a black field $2^{\mathrm{O}}$ in diameter, is raised by presenting a weak luminous field in the periphery, and this effect is not due to scattered light; the luminous surround falls almost entirely outside the summation pool yet raised the threshold. (c) The threshold for a test flash may be raised by a second flash presented not only outside the summation pool but $50 \mathrm{msec}$. later (Alpern, 1965).

It is possible that interaction between ganglion cells at the retina could explain these effects, and Rushton (1965b) suggests that the criterion for the "summation pool" is determined by a larger retinal "adaptation pool." However, when a stimulus is presented to corresponding locations on the two eyes the threshold is lower than when it is given to one eye alone. When the two stimuli occur at an interval greater than about $100 \mathrm{msec}$. the fall in threshold can be accounted for by probability summation: the increased rate of detection is that which would be given by combining two independent detectors. But when the stimuli are given at shorter intervals the fall in threshold is greater than can be accounted for by this, indicating that messages from the two eyes, each of which would be insufficient by itself to determine a positive response, may be combined and then exceed the criterion (Matin, 1962). The interaction between the eyes in dark adaptation also supports this conclusion (Wolf \& Zigler, 1955).

Thus, as with brightness averaging (Levelt, 1965a, 1965b), it appears that outputs from the two eyes are conveyed centrally, and that they are there summed and compared with the criterion. That the threshold response is selected centrally is also supported by the evidence that stimuli in one modality can affect thresholds in another (Treisman, 1964b). It follows that our overall transducer function, $E=f(I)$, must be taken to cover not only transformations occurring in the retina but also the message in the optic nerve.

(3) The transducer function: further evidence. At the retina a large number of receptors may converge on a ganglion cell, from which arises a single optic nerve fiber. We have noted that Ricco's law applies up to a limit, $\alpha$, which we can consider as a minimum sampling area for the visual system. It is likely that this can be identified with the central area of the ganglion cell receptive field, in on-center fields the area within which stimulation is excitatory (Pirenne, 1956; Glezer, 1965). We will also take $\tau$, the limit to Bloch's law, as the sampling moment or shortest duration over which the input to the retina is sampled (Crawford, 1947; Barlow, 1957; Stroud, 1955; Matin, 1962; White, 1963; Shallice, 1964; Piéron, 1965). In the model, as it has been described above, we have limited our consideration to the case where the area of the increment $\left(\mathrm{A}_{2}\right)$ coincides with that of the background $\left(A_{1}\right)$, which is assumed equal to ${ }^{\alpha}$; similarly we have taken $t_{2}=t_{1}=\tau$ 。 It can also be applied to the case where $\mathrm{A}_{2}=\alpha$ and $t_{2}={ }^{\tau}$, but the background to which this increment is added is larger and of longer duration; here, as a simplification, we assume that when the increment is 
given the visual system correctly samples the retinal area and duration occupied by it to determine the value of the central input $\mathrm{E}\left(\mathrm{x}_{5}\right)$ on that trial, and that the $\mathrm{E}_{1}$ distribution describes the inputs which are given by "sampling units" of the same area and duration as the increment, but excited by intensity $\mathrm{I}_{1}$ alone. The effect of varying $A_{2}$ and $t_{2}$ is examined below, but, at the moment, we are concerned with the model as it might apply when only a single receptive field provides the information which determines the threshold response. In this case, what neural changes take place in the visual pathway?

Since the input to a receptive field is summed at the ganglion cell we can consider two transductions: that involved in conveying the information generated by quantal absorptions to the ganglion cell, and the transformation of this into the message in the optic nerve fiber. In considering the neurophysiological evidence there is a serious difficulty, over and above that arising from the paucity of relevant information. This is that the transducer functions we are concerned with in the model may correspond to a succession of physiological transformations. Consider an input, $A_{i}$, at a point $A$ in the nervous system which is conveyed to a point $B$, where it gives rise to an output, $\mathrm{B}_{\mathrm{O}}$. The overall transducer function might be linear, $B_{O}=A_{i}$; but this could correspond to an initial logarithmic transformation, giving a message, $\log A_{i}$, which is conveyed along the channel, followed by a final exponential transformation, $B_{0}=e^{\log A_{i}=A_{i}}$. (Or we might have $B_{o}=e^{n \log A_{i}}=A_{i}^{n}$, giving an overall transduction described by a power function.) In this case, neurophysiological evidence might reveal the logarithmic relation between the message in the channel and the original input, but this would be misleading if mistaken for the overall transducer function. A related difficulty is that a physiological observation may refer to the "wrong" neural coding and thus give misleading information about the function observed. In the model we have assumed that the sensory message consists of a number of nerve impulses, the variance of each message, $\sigma_{\mathrm{S}}^{2}$, being constant, and the impulses combining additively to give $\mathrm{E}$; but, if this model applies, it might prove to be peak firing rate, minimum inter-pulse interval or some other aspect of the neural response that has the properties assigned to $s$ in the model, rather than average firing rate. If we select the wrong neural correlate of the message, the function describing it will be correspondingly misleading.

(i) Transformations in the retina.

The most important neurophysiological evidence at this stage is the observation that when the discharge from single ganglion cells in the retina of the cat or frog is recorded, and a constant threshold or suprathreshold discharge from the ganglion cell is used as criterion, then Ricco's law (complete spatial summation) is found to hold as the area of the stimulus is varied, provided it falls in the central excitatory region of a receptive field
(Hartline, 1940; Barlow, 1953; Barlow, FitzHugh \& Kuffler, 1957). Thus for different stimulus a reas the total quantity of light, $Q$, required to evoke a constant response from the ganglion cell remains constant, and this occurs at levels of illumination at which each receptor must absorb a number of quanta. The implications for some possible transformations describing the transmission from receptor to ganglion cell will be briefly considered.

(a) Logarithmic transduction. If we describe the output from a single receptor cell (as it is received at the ganglion cell) as $\mathrm{O}_{r}$, and assume that this receptor absorbs $Q$ quanta, within a sufficiently short period of time, then the output which reaches the ganglion cell might be given by $O_{r}=\log _{10} Q$ (Rushton, 1961). Thus if $Q=100$ quanta, we would have $O_{r}=2$. Let us suppose that this quantity, acting on the ganglion cell, is just sufficient to cause it to fire, i.e., the ganglion cell-firing threshold is equal to 100 quanta when these are absorbed by one receptor. If the incident quanta are now spread evenly over $n$ receptors $(n>1)$ then the output from any one of these receptors would be $\mathrm{O}_{\mathrm{r}}=\log (\mathrm{Q} / \mathrm{n})$, and the total effect on the ganglion cell will be given by ${ }_{\Sigma}^{n} O_{r}=n \log (Q / n)$. If we must have ${ }_{\Sigma}^{\mathrm{n}} \mathrm{O}_{\mathrm{r}}=2$ to reach threshold, what quantity of light will be needed to evoke the constant ganglion cell response as we vary $n$ ? Ricco's law implies that the total quantity of light, $Q_{n}$, required when $n$ receptors are stimulated, is always equal to $Q$, the quantity required for 1 receptor. But if we assume that $O_{r}=\log Q$ and take ${ }^{n} O_{r}=2$ as the threshold input to the ganglion cell, then we would have for $n=1, Q=100$; for $n=2$, $\mathrm{Q}_{2}=20$ (since $2 \log (20 / 2)=2$ ); for $\mathrm{n}=4, \mathrm{Q}_{4}=12.65$; $Q_{5}=12.56 ; Q_{8}=14.22 ; Q_{10}=15.85 ; Q_{20}=25.19 ; Q_{50}=$ $54.83 ; Q_{100}=104.7 ; Q_{1000}=1005$. These figures are meant only as an illustration, but they serve to show that a logarithmic transduction at the retinal level would not be compatible with Ricco's law. We see that initially as $\mathrm{n}$ increases instead of simple complete summation there is marked enhancement, $Q_{n}$, the total quantity of light required, falling to a minimum for 5 receptors. But then, as $n$ increases further, $Q_{n}$ also increases and summation soon falls to a very low level, with threshold intensity $\left(Q_{n} / n\right)$ almost constant. The minimum appears later for higher threshold values but the general picture remains the same: if we take $\stackrel{\mathrm{n}}{\Sigma} \mathrm{O}_{\mathrm{r}}=6$ as the critical value required to evoke a given constant response from the ganglion cell we have for $n=1, Q=10^{6}$, followed by marked enhancement as $n$ increases. The minimum is at $n=14$, when $Q_{14}=37.5$. Then there is the same rapid disappearance of summation: $Q_{100}=114.8, Q_{1000}=1014$.

It appears that a logarithmic function is difficult to reconcile with experiment. The initial enhancement could well have been missed, since it covers so small an area, but the rapid disappearance of summation beyond this small area is inconsistent with the finding that Ricco's law holds in the central receptive field. 
Glezer (1965) believes that "the excitation is ... directly proportional to the logarithm of acting light" and recognizes that "If this is the case, then increase of stimulus area at constant light intensity must cause an increase of response, i.e. an enhancement." $\mathrm{He}$ suggests "Then the law of full summation ... can be explained by the inhibitory process that arises with the spatial gradient .... The inhibition arising in the whole receptive field but with different strengths at different points. It is more strongly pronounced in the periphery of the field." It is possible to conceive an - ase of inhibition as we pass from the center of the ield (assuming that our stimulus is always at $\therefore$ center of a field) which would exactly counterbalance the enhancement produced by the logarithmic transformation, so that the excitatory and inhibitory effects together would produce an overall linear transduction from receptor to ganglion cell. But if this were the case it would appear rather surprising that the slopes of complete summation curves are so similar while the slopes of partial summation curves show considerable variation (Barlow, 1958), and since lateral inhibition decreases during dark adaptation (Barlow, FitzHugh, \& Kuffler, 1957) we would expect dark adaptation to alter the balance between the two processes and so "reveal" the underlying enhancement, which does not happen. But the major difficulty with this argument is that it rests on the assumption that the enhancement is shown for all values of $n$, which, as we have seen, is not the case. Even if inhibition ceased sharply outside the small area of enhancement, the logarithmic function would still produce the rapid disappearance of summation beyond this area, rather than Ricco's law, so that this suggestion gets us no further. Nor would it explain why, if we vary the duration of the stimulus, we do not observe short-term enhancement, followed by rapid disappearance of summation, which, rather than Bloch's law, the logarithmic transduction would produce.

(b) $\mathrm{O}_{r}=\mathrm{Q}^{1.4}$. If $\mathrm{Q}=100$ quanta are absorbed by a single receptor, this transduction would give $O_{\mathrm{r}}=631$. If we take this figure as the threshold criterion, and vary $n$, the number of receptors on which the light falls, then at threshold $631=\sum_{\Sigma}^{n} O_{r}=n\left(Q_{n} / n\right)^{1.4}$. It follows that we would get for $n=1, Q_{1}=100 ; Q_{2}=122$; $Q_{10}=192 ; Q_{100}=373$. There is no area of complete summation at all; Ricco's law should never be shown. Similarly, if $\mathrm{n}$ is taken to refer to the temporal parameter, Bloch's law would not be found.

(c) $\mathrm{O}_{r}=\mathrm{Q}^{0.3}$ If $\mathrm{Q}=100$ quanta are absorbed by a single receptor and we take $O_{r}=100^{0.3}=3.981$ as the threshold criterion, then, as we increase $n$ we get, for the threshold quantity of light, for $n=1, Q=100$; $Q_{2}=19.86 ; Q_{10}=0.46$. Enhancement begins immediately and is marked for all values of n; Ricco's law and Bloch's law should not be shown. Transductions much closer to linearity but with exponents still less than one, all show a considerable degree of enhancement.
For example, for $O_{r}=Q^{0.9}$ we would have $Q_{1}=100$, $\mathrm{Q}_{2}=92.6, \mathrm{Q}_{10}=77.4, \mathrm{Q}_{100}=60.0$.

(d) The linear function; $\mathrm{O}_{r}=\mathrm{Q}$. For any value of $\mathrm{n}_{s} \stackrel{n}{\Sigma} \mathrm{O}_{\mathrm{r}}=\mathrm{n}(\mathrm{Q} / \mathrm{n})=\mathrm{Q}$. Thus as area (or time) increases, a constant value of $Q_{n}$ will give a constant summed effect on the ganglion cell, and Ricco's law will hold until the boundary of the central excitatory field is reached. We see that this only occurs if we assume a linear transduction, and since Ricco's and Bloch's laws are such reliable experimental findings, and occur at intensities at which each receptor must absorb many quanta, it follows inescapably that a plausible model must assume linear transduction from receptor to ganglion cell. For this reason, taking the model as we have given it above as a description of the succession of events that might occur in a single receptive field and its central connection, the different transducer functions were applied not to the quanta absorbed by each receptor but to $x_{3}$, so that the latter represents a sum of linear effects of all the quantal absorptions occurring in the receptive field.

(ii) The ganglion cell output. The rate of firing in the optic nerve fiber of the horseshoe crab is approximately logarithmically related to the total light energy acting on the ommatidium (Hartline, 1934; Rushton, 1961; Ratliff, 1965), but there is little evidence on the form of the optic nerve discharge in the mammalian retina.

However, it is of interest to note that FitzHugh (1957) recorded discharges from cat ganglion cells when brief near-threshold flashes were presented against backgrounds of weak or zero intensity to which the eye was adapted. He found that the relation between the number of impulses recorded from an optic nerve fiber during a critical period following a flash, and the intensity of the latter, was usually described by a power function. The range of mean exponents for different cells examined was $0.6-1.2$.

This result would accord with a linear overall transducer function, $E=I$, if we are correct in supposing that the sensory message is coded as a number of nerve impulses. In view of the difficulties in relating neurophysiological observations of this sort to the model discussed above, it seemed desirable to examine the implications of a range of possible transductions which might relate the summed linear function of absorptions (modified by adaptation) acting on the ganglion cell to the final effect at the locus at which the threshold response is selected.

(4) Some predictions from the model.

The model has been designed to specify all the major steps which might lie between presentation of the stimulus and selection of the response (assuming that the selection process completely determines the overt response) with sufficient precision to allow predictions to be made about the size of the threshold. The variables of particular interest are the background intensity, $\mathrm{I}_{1}$, and the area $\left(A_{2}\right)$ and duration $\left(t_{2}\right)$ of the stimulus 
increment.

We have seen that $\Delta I=I_{2}-I_{1}, I_{2}$ depends on $E_{c}$, and $\mathrm{E}_{\mathrm{C}}=\mathrm{E}_{1}+\mathrm{z} \sigma_{\mathrm{E}}$ (see Fig. 1). It follows that the Weber function, the relation found between $\Delta I$ and $I_{1}$ as the latter varies, depends on the overall transducer function relating $\mathrm{E}$ and $\mathrm{I}$, and on the relation between $\sigma_{E}$ and $E_{1}$. For the linear transducer function and $N$ adaptation, the expected value of $E_{1}$ is given by $E_{1}=s g\left(I_{1}+I_{n}\right)$, which is the product of two variables. In general the variance of $\mathrm{AB}$, where $\mathrm{A}$ and $\mathrm{B}$ are two independent variables, is given by $\overline{\mathrm{A}}^{2} \sigma_{\mathrm{B}}^{2}+\overline{\mathrm{B}}^{2} \sigma_{\mathrm{A}}^{2}+\sigma_{\mathrm{A}}^{2} \sigma_{\mathrm{B}}^{2}$. Since $s$ and $g\left(I_{1}+I_{n}\right)$ are independent and the variance of the latter is described by a Poisson distribution, if we take the mean correlation between pairs of sensory messages to be $r=+1$, then the variance of $E_{1}$ is given by

$$
\sigma_{E}^{2}=\sigma_{s}^{2} g^{2}\left(I_{1}+l_{n}\right)^{2}+s^{2} g\left(1_{1}+l_{n}\right)+\sigma_{s}^{2} g\left(I_{1}+I_{n}\right)
$$

We can consider the first term on the right as determined by the sensory noise, the second by the physical noise.

Since $E_{2}-E_{1}=E_{C}-E_{1}=2{ }^{\sigma} E=s g\left(I_{2}+I_{n}\right)-s g\left(I_{1}+I_{n}\right)=$ $\mathrm{sg} \Delta \mathrm{I}$, it follows that the Weber function is given by

$$
\Delta l=\frac{z}{s g} \cdot \sigma_{E}=\frac{z}{g} \sqrt{g^{2} v^{2}\left(\left.\right|_{1}+\left.\right|_{n}\right)^{2}+g\left(\left.\right|_{1}+l_{n}\right)+v^{2} g\left(l_{1}+l_{n}\right)}
$$

where $v=\sigma_{\mathrm{S}} / \mathrm{s}$. This relation contains four parameters, $z, g, v$, and $I_{n}$, but independent procedures for estimating them can be designed (Treisman, 1964a). If $\mathrm{v}^{2}<<1$, which is plausible, then for $\mathrm{g}\left(\mathrm{I}_{1}+\mathrm{I}_{\mathrm{n}}\right)$ small the function will approximate to

$$
\Delta I=z^{-1 / 2}\left(I_{1}+l_{n}\right)^{1 / 2}
$$

This is the square root law which has been predicted from the quantum fluctuations of light (Rose, 1948; Barlow, 1957). Equation (2) shows that when the number of quanta absorbed is small the threshold is mainly determined by the effects of the physical variability of light, and the "dark light" due to spontaneous firing, but the effect of the sensory noise arising from the variability of central transmission of messages is negligible. This accords with the relatively close approach of brightness discrimination thresholds at low intensities to the limits set by the physical nature of light (Hecht, Shlaer, \& Pirenne, 1942; Crouzy, 1961).

The square root law has been found to hold for low intensities of I but not for high (Barlow, 1957). "'When the test stimulus is of long duration or large area, and when the background intensity is high" (Barlow, 1957) "the experimental points deviate from the appropriate theoretical curve and tend to obey the Weber law instead" (Barlow, 1958). The reason for this is immediately apparent from equation (1); an increase in any of these three variables will increase $g\left(I_{1}+I_{n}\right)$, (since $g=f A t$ ), and when this quantity is large the correlated component of the variance of $E$, which arises from the sensory noise, becomes predominant and equation (1) approximates to

$$
\Delta I=\mathbf{z v}\left(\left.\right|_{1}+I_{n}\right)
$$

the linear generalization of Weber's law. Thus the model predicts both the square root law found experimentally at low intensities, and the continuous transition to the traditional Weber function which is observed at high intensities.

If we assume $\mathrm{C}$ adaptation, and $\mathrm{O}<\mathrm{r}<1$, equation (2) remains unchanged, but equation (3) becomes

$$
\Delta !=\operatorname{zver}^{1 / 2}\left(I_{1}+I_{n}\right)
$$

Thus a low value of $\mathrm{c}$ or $\mathbf{r}$ will imply a low value of $\Delta I$ if $I_{1}$ is large, but will have little effect on the threshold when $I_{1}$ is small. One consequence is that if either $c=0$, as in $M$ adaptation, or $r=0$, the term in equation (1) which bulks large when $g^{2}\left(I_{1}+I_{n}\right)^{2}$ is large, and so determines the transition to Weber's law (equation (4)) disappears, and the square root law will now hold for the whole range of values of $I$.

We can discover what the consequence of varying the area or duration of the increment will be by writing $g$ in full in equation (1). The case we have considered so far is that in which the increment coincides with a "sampling unit" of area $\propto$ and duration $t$, but if we vary $A_{2}$ or $t_{2}$ other cases will arise. The simplest assumption to make is that in order to compute the $\mathrm{E}_{1}$ distribution and so the best location of $E_{c}$, when the background is illuminated by $I_{1}$, the visual system records the values of $\mathrm{E}$ given by samples whose area and duration equal those of the increment, (i.e., it takes $A_{1}=A_{2}$ and $t_{1}=t_{2}$ ) when $A_{2} \geq \propto$ and $t_{2} \geq \tau$. When $A_{2}<\propto$ the visual system must, of course, take $A_{1}=\alpha$, the minimum sampling area, and similarly when $\mathrm{t}_{2}<\tau, \mathrm{t}_{1}=\tau_{0}$

Since $E_{c}-E_{1}=s\left[g_{1}\left(I_{1}+I_{n}\right)+g_{2} \Delta I\right]-s_{1}\left(I_{1}+I_{n}\right)=$ $\mathrm{sg}_{2} \Delta \mathrm{I}=\mathrm{z} \sigma_{\mathrm{E}}$ (see Fig. 1), we can write equation (1) as

$$
\Delta I=\frac{z}{f A_{2}{ }^{+} 2} \sqrt{f^{2} A_{1}^{2} t_{1}^{2} v^{2}\left(1, l_{n}\right)^{2}+f A_{1} t_{1}\left(1+v^{2}\right)\left(I_{1}+l_{n}\right)}
$$

This approximates to

$$
\Delta I=\frac{z A_{1}^{1 / 2} t_{1}^{1 / 2}\left(I_{1}+I_{n}\right)^{1 / 2}}{f^{1 / 2} A_{2} t_{2}}
$$

when $g_{1}\left(I_{1}+I_{n}\right)$ is small, and, when this quantity is large, to

$$
\Delta I=\frac{A_{1}^{\dagger} l^{z v}\left(l_{1}+I_{n}\right)}{A_{2}{ }^{\dagger} 2}
$$




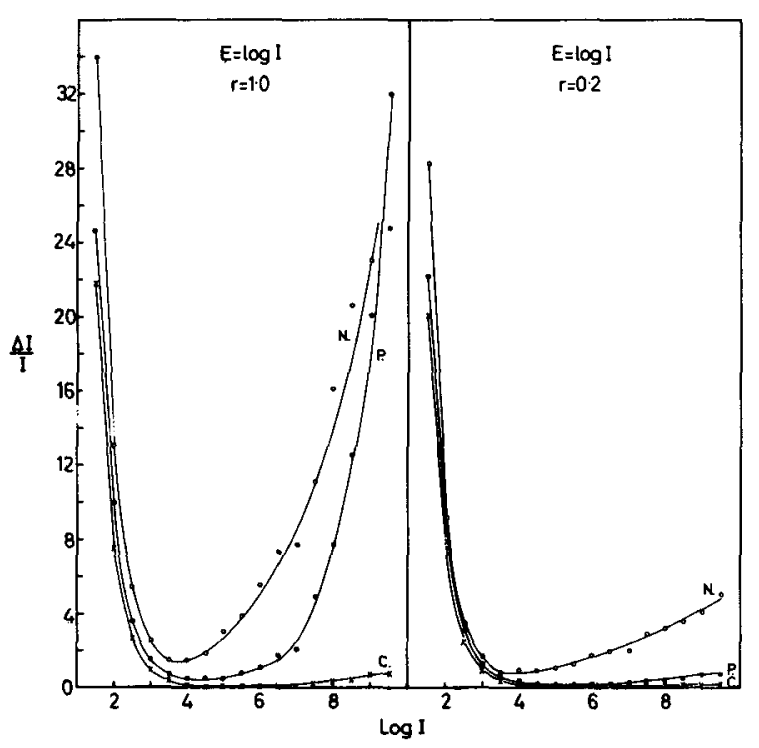

Fig. 2. The Weber fraction, $\Delta \mathbf{I}_{\mathbf{I}_{1}}$, is plotted against $\log \mathbf{I}_{1}$. The transducer function was $E=\log I$, and $r=1.0$ or 0.2 . The Weber function is shown for three conditions of adaptation, $N, P$ and $C$.

Equations (6) and (7) become equations (2) and (3) when $A_{1}=A_{2}$ and $t_{1}=t_{2}$.

Let us suppose that $I_{1}, A_{1}$ and $A_{2}$ are constant and that $t_{2}$ varies. For $t_{2}<\tau, t_{1}=\tau$, so that all terms on the right-hand side of equation (5) will be constant except $t_{2}$, and the equation can be written $\Delta I=k / t_{2}$, or $\Delta I t_{2}^{1}=k$, where $k$ is a constant. This is Bloch's law of complete temporal summation. It is determined by the assumptions that there is a minimal period below which reduction in the duration of the increment does not reduce the duration of background noise sampled (Brindley, 1960), and that the effects of quantal absorptions falling within the minimum period do not undergo any non-linear transformation before they are summed.

For $t_{2}>\tau$ and variable either equation (6) or equation (7) may hold. Since now $t_{1}=t_{2}$, equation (6) will simplify to $\Delta \mathrm{I}=\mathrm{k}^{\prime} / \mathrm{t}_{2}^{1 / 2}$, or $\Delta \mathrm{It}_{2}^{1 / 2}=\mathrm{k}^{\prime}$, where $\mathrm{k}^{\prime}$ is a constant. Thus for durations of the stimulus increment which exceed the limit for complete summation the difference threshold (or absolute threshold, $I_{0}$, if $I_{1}=0$ ) is now inversely related to the square root of the duration of the stimulus increment. But this relation holds only when $g_{1}\left(I_{1}+I_{n}\right)$ is small. If $I_{1}$ or $A_{1}=A_{2}$ is sufficiently large, or, if these are small, when $t_{1}=t_{2}$ becomes large, the relation between threshold and duration will approximate to that described by equation (7). As $t_{1}=t_{2}$, all the terms on the righthand side of this equation are constants, so that it can be written $\Delta I=k^{\prime \prime}$ or $\Delta I t_{2}^{0}=k^{\prime \prime}$, where $k^{\prime \prime}$ is a constant: summation is no longer shown. Thus the model predicts a transition from complete summation to a square root law, and that the exponent of $t_{2}$ will then decrease from 0.5 to zeroas $I_{1}, A_{2}$ or $t_{2}$ increase.

This decrease in summation is not attributed to the classical assumption that some decline in the efficiency with which the stimulus is processed occurs as its duration (or area) is increased and we do not need to suppose that the visual system fails to extract any further information when the stimulation exceeds an arbitrary "utilization time" (Piéron, 1965) or limiting area. Instead we assume that whatever the area or duration of stimulation maximum use is made of all the information available. Complete summation reflects the existence of a minimum sampling time. For intervals greater than $\tau$ the exponent of $t_{2}$ declines because of the limits on discrimination imposed by the variability of the central effect of the stimulus. Initially the Poisson fluctuation of light predominates in determining this noise, giving a square root law; but because positively correlated sensory noise becomes predominant when the total input from the stimulus is large, as the intensity, area or duration become high the exponent declines from 0.5 to 0 .

The predicted forms of the Weber function, and of the functions for spatial and temporal summation, agree with many experimental findings. However, these predictions are based on a limited set of assumptions, such as that $E=I$ and $r=+1.0$; the computations presented below were designed to show how far the predictions would be maintained when the main assumptions of the model were varied.

\section{The form of the Weber function.}

Values of $\Delta I$ were computed for different intensities of $I_{1}$, conditions of adaptation, transducer functions, and levels of correlation. Some of the resulting Weber functions are shown in Figs. 2, 3 and 4. In these the Weber fraction, $\Delta I / I_{1}$, has been plotted against $\log I_{1}$, as is often done in practice. In each figure Weber functions are shown for N, P and C adaptation, and for

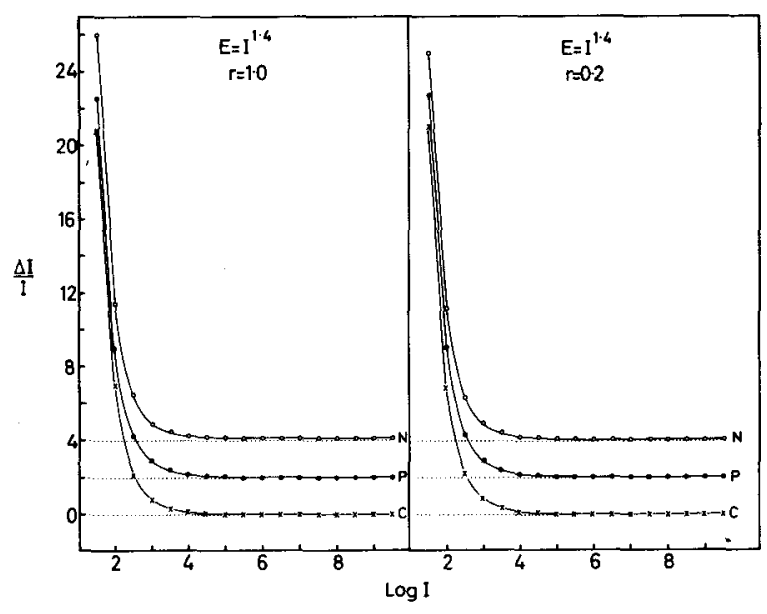

Fig. 3. As Figure 2, except that $E=I$ 1.4. The $N$ adaptation curve is here shifted upwards 4 units, and the $P$ curve is shifted up $z$ units. 


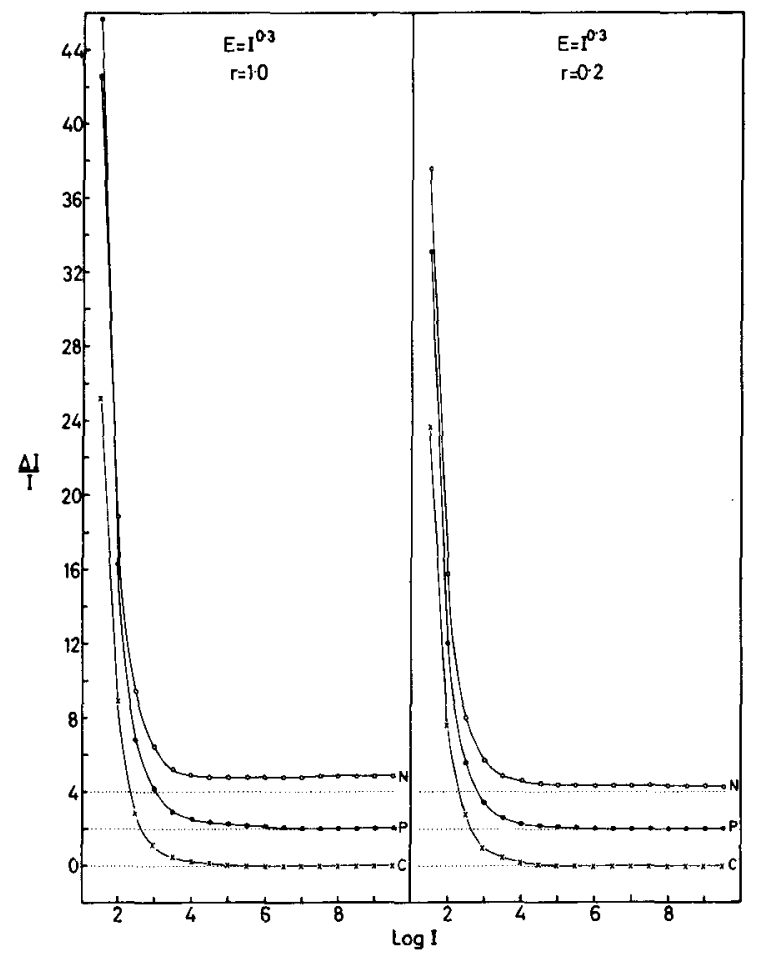

Fig 4. As Figure 3, except that $E=\mathbf{1}^{0.3}$.

$r=1.0$ and $r=0.2$. The three transducer functions shown are $E=\log I, E=I^{1.4}$ and $E=I^{0.3}$. The results for $\mathrm{E}=\mathrm{I}$ and $\mathrm{E}=\mathrm{I}^{0.6}$ were intermediate between those shown in Figs. 3 and 4 . The curves in Figs. 3 and 4 , but not in Fig. 2, are staggered. The effective zero for each curve is shown by a dotted line.

We see that all the power functions examined give Weber functions which appear acceptably similar to those obtained experimentally, for all the combinations of adaptation and correlation examined, but this is not the case for $E=\log I$. For the logarithmic transducer function, as for the power functions, the thresholds fall as the degree of adaptation increases and as the level of correlation decreases, but, unlike the power functions, after first falling to a minimum, the Weber fraction shows a continuous rise over the remaining range of values of $I_{1}$. A rise in the Weber fraction, starting at a high intensity level, has sometimes been found experimentally (Steinhardt, 1936; Holway, 1937; Pirenne, 1962a), but it is suppressed if precautions are taken to ensure that light adaptation is fully maintained when $I_{1}$ is high. Adaptation was, of course, maintained at a constant level for each curve in Fig. 2. Aguilar and Stiles (1954) found that the rod mechanisms became saturated (i.e., $\Delta I$ I I rose) at high levels of stimulation. But this was a phenomenon of sharp onset occurring at a level of illumination at which we can suppose discrimination would normally be produced by the cones, and for a considerable range of intensities below this a linear Weber function was shown. Although our results do not immediately exclude the logarithmic transducer function they suggest that it is unlikely to give a more or less acceptable Weber function unless a high degree of adaptation and a very low level of correlation can be assumed. The effects of threshold size are shown moreclearly in Figs. 5 and 6 , which give $\Delta I$ for each transducer function, $r=1.0,0.6$ or 0.2 , and $\mathrm{N}, \mathrm{P}$ or $\mathrm{C}$ adaptation. In Fig. $5 \Delta \mathrm{I}$ is in each case the average for $I_{1}=10^{1.5}$, $10^{2}, 10^{2.5}$ and $10^{3}$ quanta/sec. deg. ${ }^{2}$ (these all gave similar values, at or close to the absolute threshold), and in Fig. $6 \mathrm{I}_{1}=10^{9}$ quanta/sec. deg。 ${ }^{2}$.

We saw earlier that, with the linear transducer function, an increase in the degree of adaptation (a fall in c), or reduction in the level of correlation, would give lower thresholds for $I_{1}$ high (this follows from equation (4)), but would have little effect for low values of $I_{1}$. The results in Fig. 5 accord with this: the values of $\Delta I$ are very similar for the three degrees of correlation and the different types of adaptation when $\mathrm{E}=\mathrm{I}^{1.0}$ (and when $\mathrm{E}=\mathrm{I}^{1.4}$ ). However, effects of these conditions are shown when the transducer function is a power law with a small exponent $\left(E=I^{0.6}\right.$ and $\left.E=I^{0.3}\right)$ or is logarithmic. Figure 6 shows that changes in the degree of adaptation or correlation produce very much greater differences in $\Delta I$, both absolutely and relatively, at high values of $I_{1}$. These differences are shown for all the functions but are greatest for power laws with low exponents and the logarithmic function.

The usual plot of the Weber fraction, $\Delta \mathrm{I} / \mathrm{I}$, against I or $\log I$, is not well adapted to show a transition between the square root and linear segments of the Weber function, as illustrated by Fig. 7, which gives calculated Weber functions for $\mathrm{E}=\log \mathrm{I}, \mathrm{E}=\mathrm{I}^{0.3}$ and $\mathrm{E}=\mathrm{I}^{1.0}$, for $\mathrm{N}$ adaptation and $\mathrm{r}=0$. Zero correlation will produce a square root law for all values of $I$, but the Weber functions for $\mathrm{I}^{0.3}$ and $\mathrm{I}^{\mathrm{l} .0}$ shown in this figure are very similar to those in Figs. 3 and 4 ,

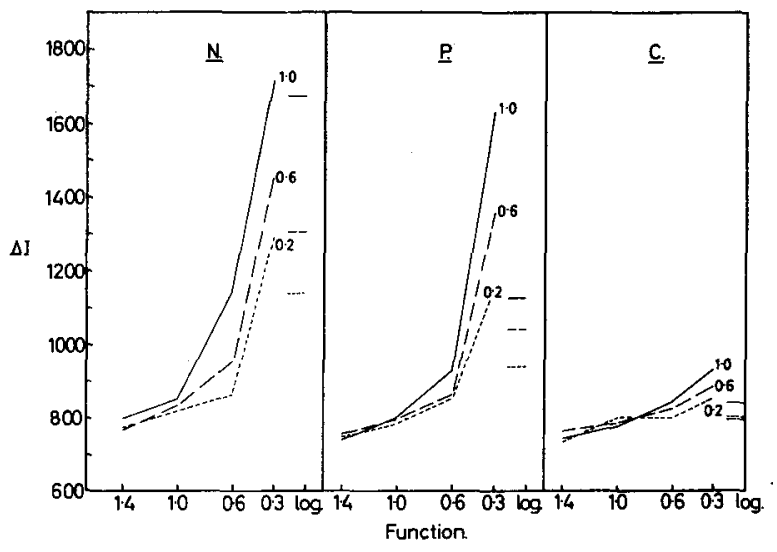

Fig. 5. Values of $\Delta I$ (in quanta/sec.deg. ${ }^{2}$ ), averaged for $I_{1}=$ $10^{1.5}, 10^{2}, 10^{2.5}$ and $10^{3}$ quanta/sec.deg. ${ }^{2}$ are shown for each transducer function, for $N, P$ and $C$ adaptation, and for $r=1.0,0.6$ and 0.2 (the values of $r$ are shown rext to the corresponding curves). 


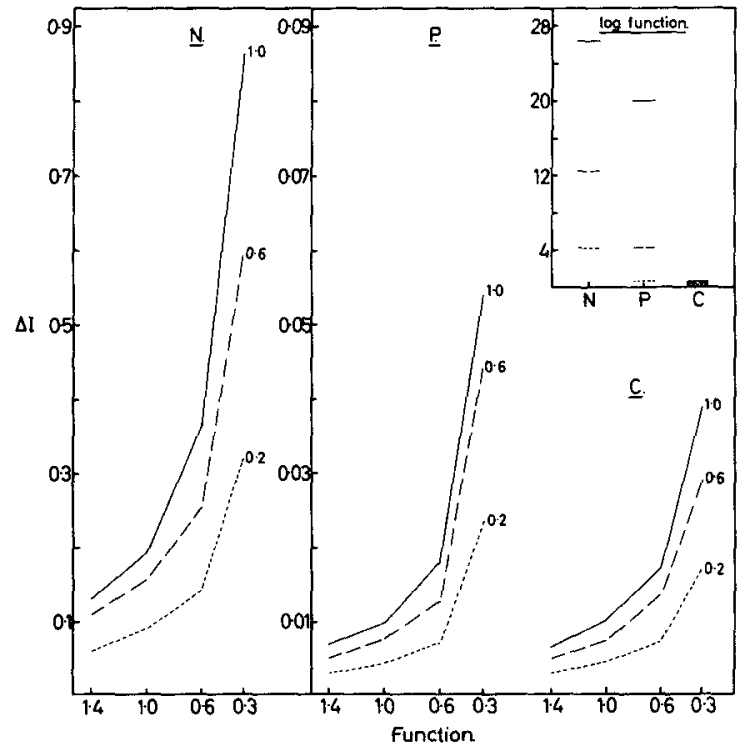

Fig. 6. $\Delta I$ (in units of $10^{9}$ quanta $/$ sec.deg. ${ }^{2}$ ) for $I=10^{9}$ quanta/ sec.deg. ${ }^{2}$ is shown for each transducer function, for $r=1.0,0.6$ and 0.2 , and for $N, P$ and $C$ adaptation. The values for the $\log$ function are shown separately.

and give the deceptive appearance of according with Weber's law when $I$ is high. It is of interest that even when $r=0$ the logarithmic function shows a low minimum followed by a continuous rise for values of I above the minimum.

The relation between threshold and intensity is shown more clearly when $\log \Delta \mathrm{I}$ is plotted against $\log \mathrm{I}_{1}$. This has been done in Figs. 8 and 9, which confirm that all the power laws examined give Weber functions whose general form is in close agreement with the usual experimental results (Aguilar \& Stiles, 1954; Barlow, 1957). The initial horizontal segment is mainly determined by $I_{n}$, the dark light, and is at the level of the absolute threshold. This is followed by a zone of transition to which a square root law can be more or less satisfactorily fitted; as I increases the exponent of the Weber function (the slope in $\log -\log$ coordinates) increases, until finally the function is linear and Weber's law at last applies. The transitions from one segment to the next appear fairly sharp for $E=I^{1.4}$ or $\mathrm{I}^{1.0}$, but are more gradual for transducer functions with a low exponent or the $\log$ function. Low values of $r$ and $c$ both prolong the square root segment and so delay the transition to Weber's law, which accounts for their effect in reducing the threshold for high values of $I_{1}$. The transition also tends to occur earlier if the exponent of a power function is low.

The logarithmic function gives a similar curve. Above the absolute threshold there is a fairly rapid transition from the square root segment to a slope which in this case exceeds one. For $r=1.0$ these slopes are $1.24(\mathrm{~N})$ and $1.20(\mathrm{C})$, for $\mathrm{r}=0.2$ they are $1.15(\mathrm{~N})$ and $1.12(\mathrm{C})$. These curves are clearly dif- ferent from those of Aguilar and Stiles (1954).

Barlow (1957) takes as an estimate of $I_{n}$ the value of I which corresponds to the point of intersection of the horizontal line through the absolute threshold values and a straight line fitted to the initial ascending limb of the Weber function, plotted as $\log \Delta I$ against $\log$ I. Figures 8 and 9 show that this will give satisfactory estimates for power functions not too far removed from linearity, but would lead to considerable underestimation if a logarithmic function or a power transducer function with a low exponent held.

Figure 10 shows functions for $E=\log I, I^{0.3}, I^{1.0}$ and $I^{1.4}$ when $r=0$. We saw earlier that if the transducer function is linear, zero correlation gives a square root law for all values of $I$, and this is shown for $E=I$ and also for $\mathrm{E}=\mathrm{I}^{1.4}$. Even with zero correlation, however, it appears that a power function with a low exponent will depart from the square root law: for $E=I^{0.3}$ the exponent of the corresponding Weber function is 0.84 when $I$ is large. For $E=\log I$ the slope of the Weber function, though less than was shown with positive correlations, is still greater than one when $I$ is large (it is 1.05). In contrast with $r=0$, computations for $\mathbf{r}=+0.01$ ( $\mathrm{N}$ adaptation) showed that all the power transducer functions gave linear Weber functions at high values of $I$; the logarithmic transducer function gave a slope a little greater than one.

The effects of the five different adaptation assumptions are compared in Fig. 11 for $E=I$ and in Fig. 12 for $\mathrm{E}=\mathrm{I}^{0.3}$, with $\mathrm{r}=0.6$. When the transducer function is linear $\mathrm{N}$ and $\mathrm{F}$ adaptation produce identical thresholds. With $C$ adaptation the linear segment of the Weber function starts later and, as implied by equation (4),

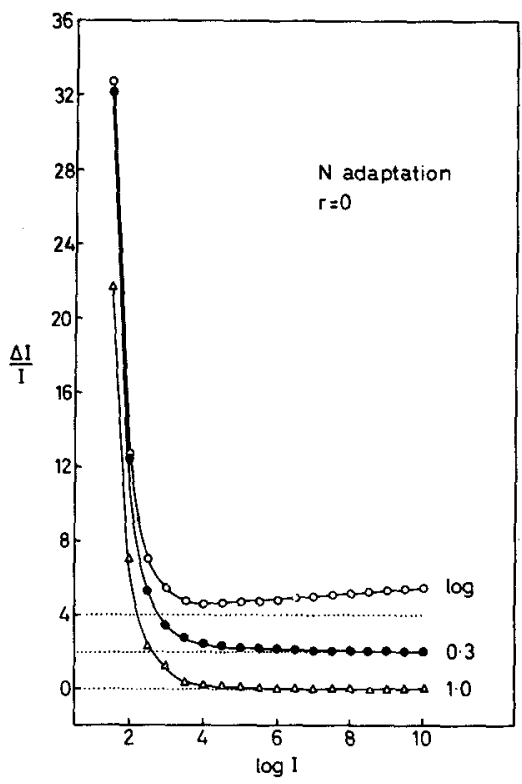

Fig. 7 . The Weber functions for $r=0$ and $N$ adaptation. $\Delta I / I$ is plotted against $\log I$ for $E=\log I$ (this curve is displaced 4 units upwards), $E=I^{0.3}$ (displaced 2 units upwards) and $E=I 1.0$. 


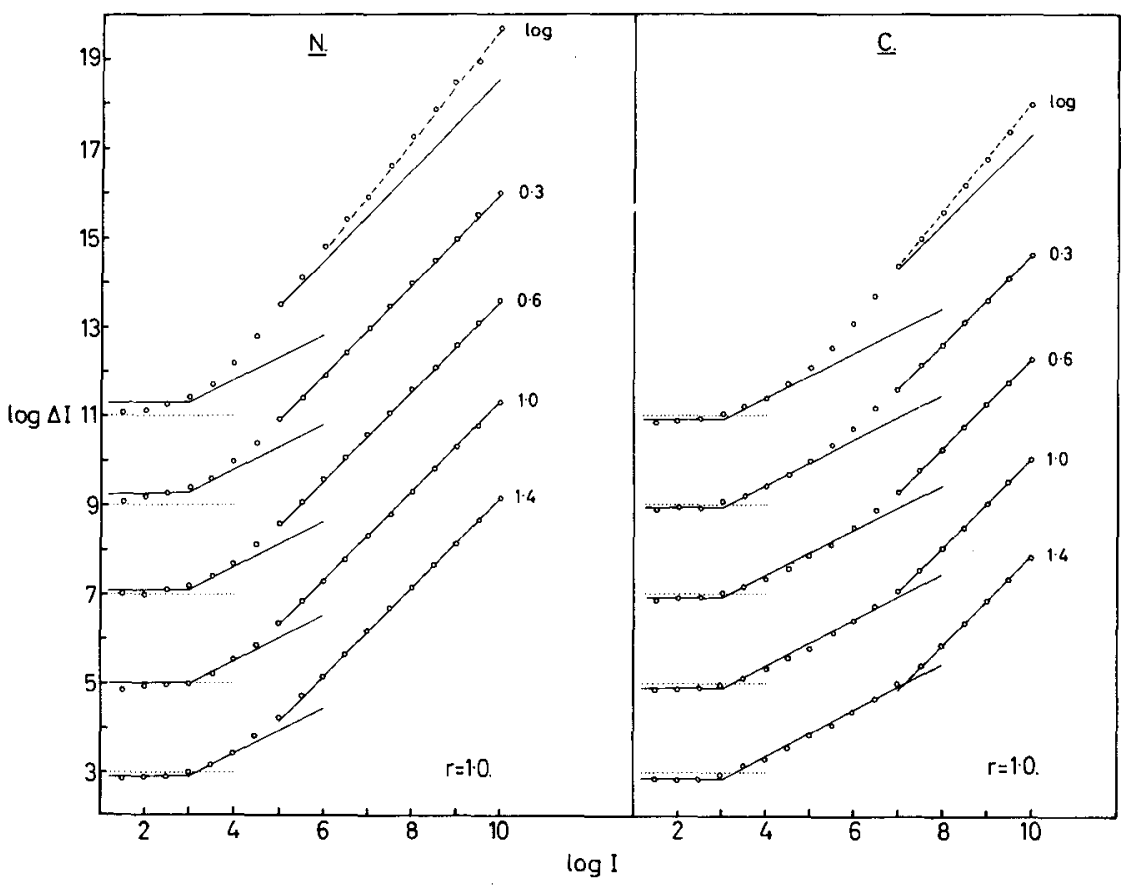

Fig. 8. Log $\Delta$ l plotted against $\log I$ for the 5 transducer functions and $\mathbf{N}$ and $\mathbf{C}$ adaptation, with $r=1.0$. The power function exponents are shown next to the corresponding curves. Each successive curve above the lowest is shifted upwards $2 \log$ units. The three continuous lines fitted to each curve (by eye) have, from left to right, slopes of 0 , 0.5 and 1.0. In each case the first two lines were made to intersect at $\log I=3$. The dashed lines fitted to the log functions have slopes of 1.24 ( $\mathrm{N}$ adaptation) and 1.20 (C adaptation). it is displaced downwards, as compared with $\mathrm{N}$ adaptation, by $\log c$ (here $c=0.05$ and the displacement is therefore $1.3 \mathrm{log}$ units). It appears that the effect of the "adaptation level" included in $\mathrm{P}$ adaptation is negligible: the curves for $P$ and $C$ adaptation were so similar that they could not be plotted separately. Finally, as expected, $M$ adaptation gives a square root law for all values of $I$. The results are similar when $\mathrm{E}=\mathrm{I}^{0.3}$ (see Fig. 12), the main difference being that
$\mathrm{P}$ adaptation now falls between $\mathrm{N}$ and $\mathrm{C}$ adaptation, tending towards the latter as I increases.

We have now seen how the predictions of the model vary as we alter our assumptions about adaptation, the transducer functions, and levels of correlation. The area and duration of the stimulus increment are two further variables which affect the threshold, and in the next section we shall examine the effects of variation in these.

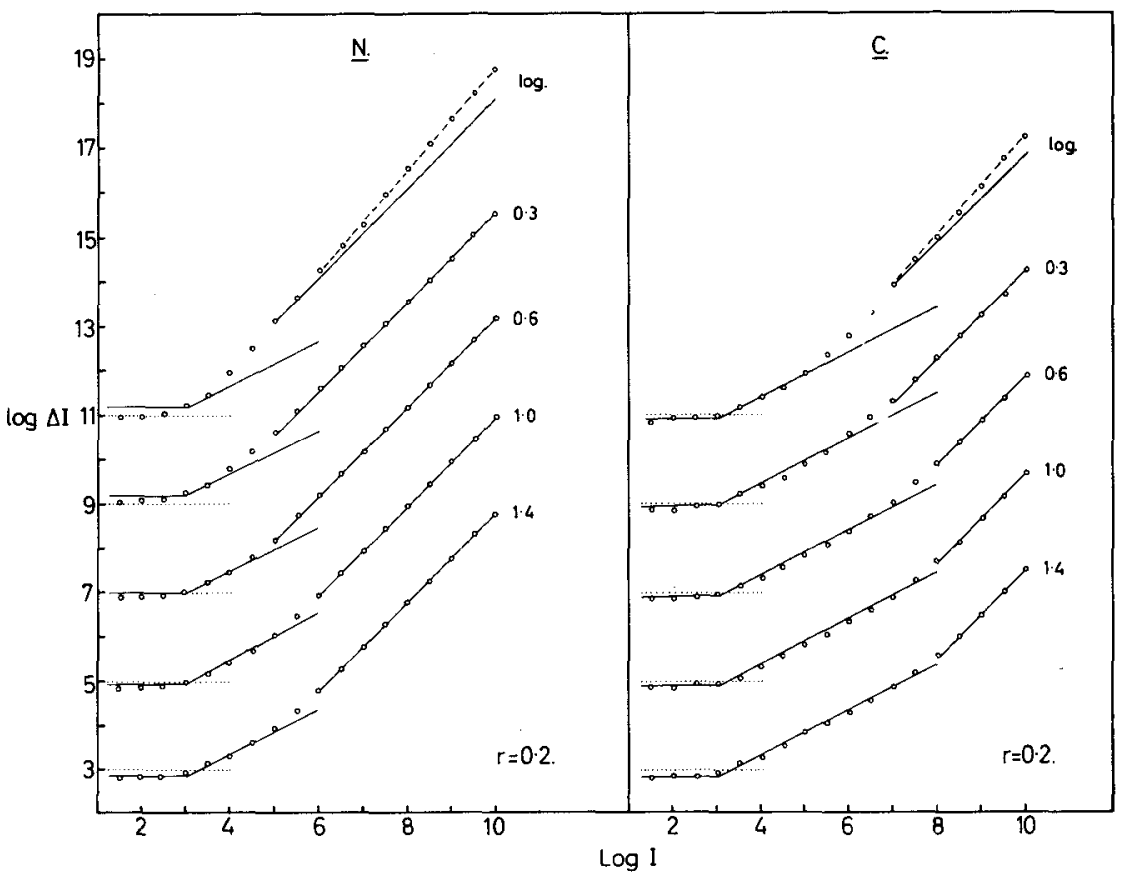

Fig. 9. As Figure 8, except that $\mathrm{r}=0.2$. The dashed lines fitted to the log functions have slopes of 1.15 ( $N$ adaptation) and 1.12 ( $C$ adaptation). 


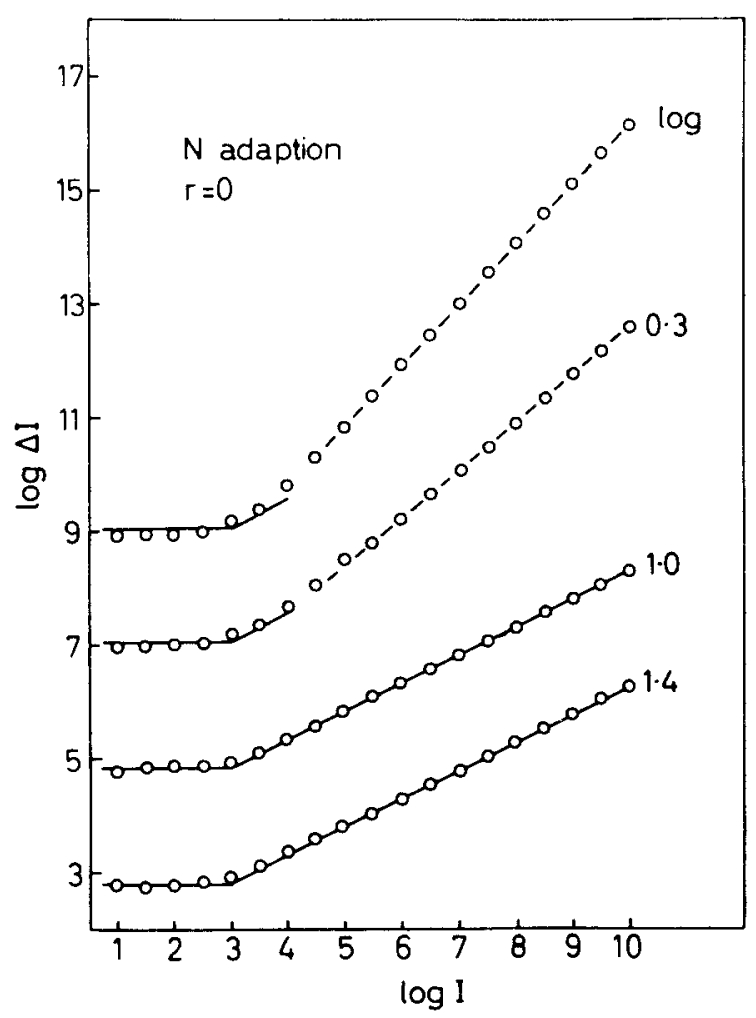

Fig. 10. $\log \Delta I$ is plotted against $\log I$ for 4 transducer functions $\left(E=\log 1 ; 1^{0.3} ; 1^{1.0} ; I^{1.4}\right)$, for $r=0$ and $N$ adaptation. Each successive curve above the lowest is shifted upwards 2 log units. The continuous lines were fitted by eye and have, from the left, slopes of 0 and 0.5 ; they were made to intersect at $\log I=3$. The dashed line fitted to the curve for $E=I^{0.3}$ has a slope of 0.84 , and that fitted to $E=\log I$ has a slope of 1.05 .

\section{Spatial and temporal summation.}

Predictions were made earlier about the threshold changes that would result when the duration and area of the stimulus increment are varied. Though it is so universally used that it could not be abandoned, the term "summation," used to describe some of these effects, is unfortunate, since it is often taken to refer not only to an observation, but also to an explanation for it. Thus the transition from "complete" to "partial summation" is sometimes understood to imply the hypothesis that when the stimulus area or duration are large a proportion of the quantal absorptions are in some way "Iost" or not taken account of, and that this is why the total quantity of light required to reack threshold rises. This is not the explanation the present model provides. We take it that complete spatial and temporal summation result from the existence of a minimum sampling area, probably based on the receptive field, and a minimum sampling duration. As we saw, this led to Bloch's law, $\Delta I t_{2}^{1}=k\left(\right.$ or $\left.\log \Delta I=\log k-\log t_{2}\right)$ for $t_{2} \leq \tau$; Ricco's law for spatial summation, $\log \Delta I=$ $\log k-\log A_{2}$, can be similarly derived. For $t_{2}>\tau$ the square root law, $\Delta \mathrm{It} \mathrm{t}_{2}^{1 / 2}=\mathrm{k}^{\prime}$, and the transition to zero summation, $\Delta \mathrm{It} \mathrm{t}_{2}^{0}=\mathrm{k}^{\prime \prime}$, are consequences of the limits to discrimination imposed by the positively correlated sensory noise, and its increasing importance in determining $\Delta \dot{I}$ or the absolute threshold as $g_{1}\left(I_{1}+I_{n}\right)$ increases. Thus the same reasons which explain the change in the exponent of $\left(I+I_{n}\right)$ from 0.5 to 1.0 as I increases, when we study the Weber function, also account for the decrease in the exponent of $t_{2}$ (or $A_{2}$, to which an exactly similar argument applies) from 0.5 to 0 , as $t_{2}$ increases. For both the Weber and summation functions the change in exponent is similarly expedited by an increase in $I_{1}, A_{2}$ or $t_{2}$.

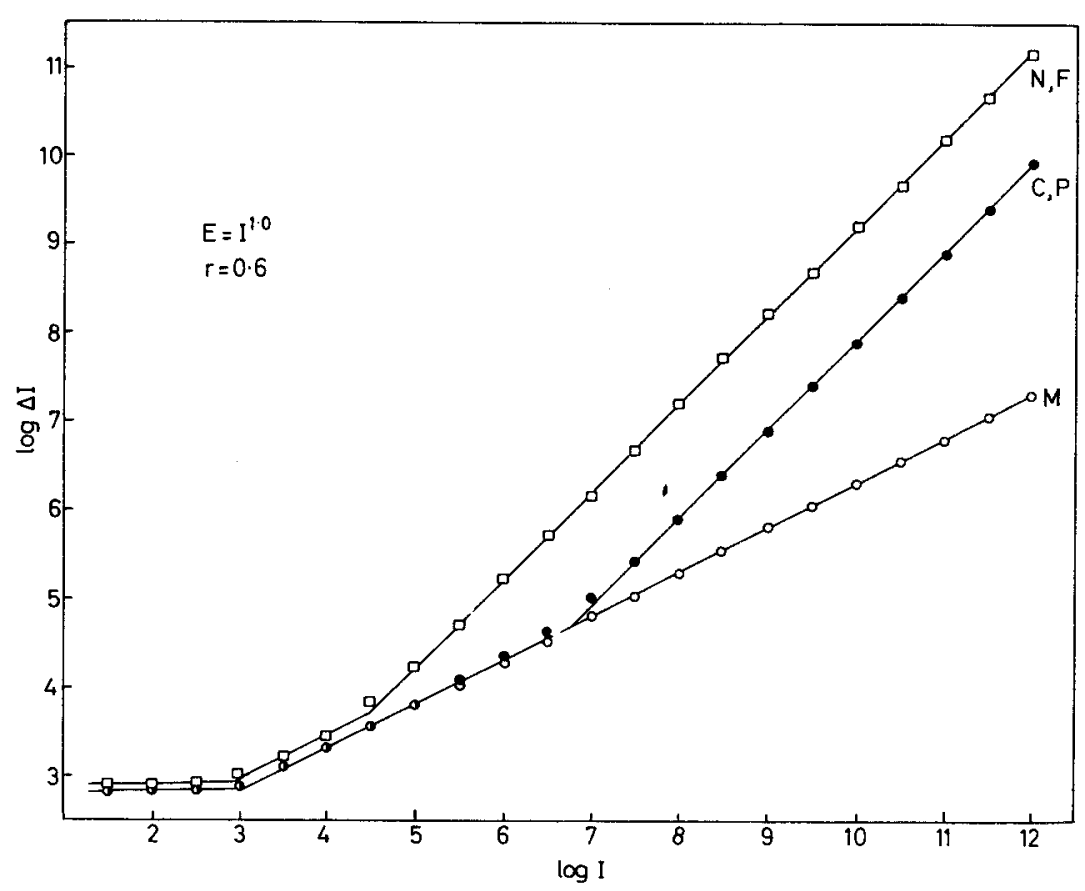

Fig. 11. Log $\Delta I$ is plotted against $\log$ $I$ for $E=I^{1.0}$ and $r=0.6$. $N$ represents no adaptation $\left(\mathrm{x}_{3}=\mathrm{x}_{2}\right)$; $F$ represents fractional adaptation $\left(x_{3}=0.005 x_{2}\right) ; P$ is partial adaptation (see text); $C$ is complete adaptation $\left(x_{3}=x_{2}-x_{1}+0.05 x_{1}\right)$ and $M$ is adaptation to the mean $\left(x_{3}=x_{2}-x_{1}\right)$. $\left(x_{1}\right.$ is the mean number of quantal absorptions per trial, $x_{2}$ is the actual number of absorptions on a given trial, and $x_{3}$ is the number of absorptions which contribute to determining the magnitude of the sensory message generated after adaptation). From left to right, the straight lines, which have been fitted by eye, have slopes of $0,0.5$ and 1.0. 


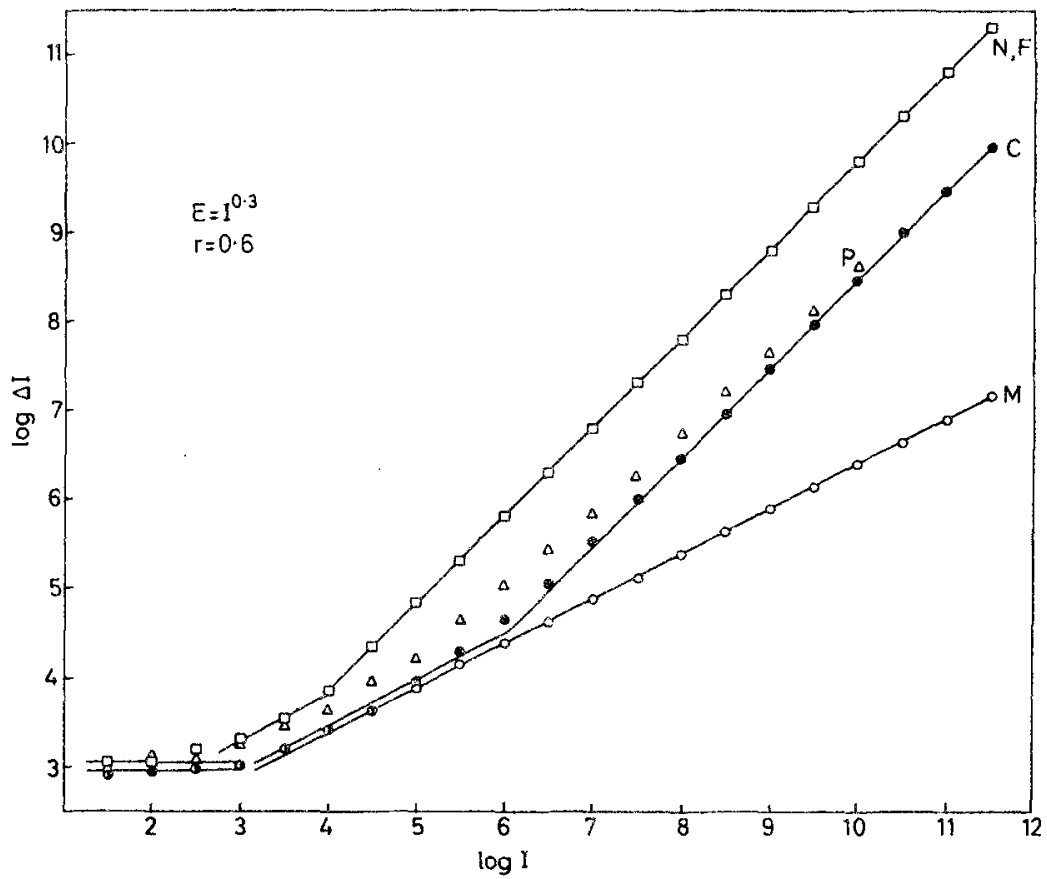

Fig. 12. As Figure 11, except that $E=I^{0.3}$. No lines have been fitted to the results for $P$ adaptation (indicated by open triangles).
Figure 13 shows temporal summation data obtained by Barlow (1958). They appear to be in full accord with the predictions from the model. As log $\Delta I$ is plotted against $\log t$, a slope of -1 corresponds to complete summation, -0.5 to the square root law $\left(\log \Delta I=\log k^{\prime}-0.5 \log t_{2}\right)$, and a zero slope indicates that there is "no summation." Examining those portions of the curves which lie beyond the range of complete summation, we see that for any value of $t_{2}$ the slope tends to be flatter if $I_{1}$, the background intensity, is high and if the stimulus area is large; and, of course, as $t_{2}$ increases the curves flatten out. Corresponding effects are shown by spatial summation curves (Barlow, 1958; Glezer, 1965). Two further features of the results in Fig. 13 are worthy of note. First, the thresholds are uniformly lower for the large stimulus area $\left(\mathrm{A}_{2}=27.6 \mathrm{deg} .{ }^{2}\right)$ than for the small $\left(A_{2}=0.011 \mathrm{deg} \cdot{ }^{2}\right)$. This would be expected when either equation (6) or (7) applies (the values of $I_{1}$ and $A_{2}$ determine whether one or other or an intermediate form holds for a given range of $t_{2}$ ). Equation (6) contains the term $A_{1}^{1 / 2} / A_{2}$. When $A_{2}$ is large $A_{2}=A_{1}$, and this term is small; thus for $A_{2}=27.6 \mathrm{deg} .{ }^{2} A_{1}^{1 / 2} / A_{2}$ $=1 / \mathrm{A}_{2}^{1 / 2}=0.19$. When $A_{2}<\propto$ (we will take $0.1 \mathrm{deg} .^{2}$ as a reasonable value for $\propto$ (Barlow, 1958)) we get a large value for $A_{1}^{1 / 2} / A_{2}$, which becomes ${ }_{\alpha} 1 / 2 / A_{2}=$ $0.1^{1 / 2} / 0.011=28.8$. Thus the change from the large to the small value of $\mathrm{A}_{2}$ would cause an increase in $\log \Delta \mathrm{I}$ of the order of $2.18 \mathrm{log}$ units, if equation (6) applies.

Equation (7) contains the term $A_{1} / A_{2}$. When $A_{2}$ is large. $A_{1} / A_{2}=1$. For $A_{2}<\propto$ this term becomes larger: $\propto / A_{2}=0.1 / 0.011=9.09$. Thus the decrease in $A_{2}$ should increase $\log \Delta I$ by about $0.96 \log$ units, if equation (7) applies.

These predicted threshold changes agree fairly well, though not exactly, with those shown in Fig. 13, and, as we would expect, the differences in the figure decrease as $I_{1}$ increases. We could not expect to predict the differences very accurately, not only because we have had to assign a somewhat arbitrary value to $\alpha$, but also because $\propto$ is not constant. A second notable

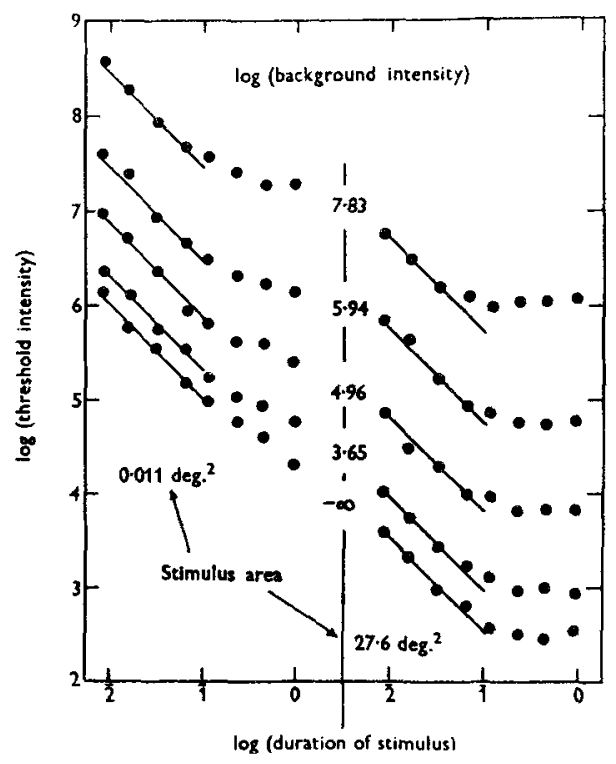

Fig. 13. Data obtained by Barlow (1958), Log $\Delta I$ (or $\log I_{o}$ ) is plotted against $\log t$ for a small $\left(0.011 \mathrm{deg}{ }^{2}\right)$ and a large (27.6 deg. ${ }^{2}$, stimulus area at five different background intensities. The straight lines have a slope of $-I$ and continue up to 0.1 sec. Intensities are given in quanta $\left(507 \mathrm{~m}_{\mu}\right) /$ sec. deg. $^{2}$, durations in seconds. 
feature of the results in Fig. 13 is that $\tau$ decreases as $A_{2}$ or $I_{1}$ increase, and this parallels a similar effect of $I_{1}$ and $t_{2}$ on $\propto$, which can be treated as constant only as a first approximation.

These changes in $~$ and $\tau$ (Keller, 1941; Barlow, 1958; Glezer, 1965; Piéron, 1965) do not follow from the model which has been presented here, but require some additional explanation. They have led Treisman (1964a) and, in more detail, Glezer (1965) to suggest that $\propto$ and $\tau$ may be controlled by feedback processes. These may serve to prevent the total output of a "sampling unit," i.e., a central receptive field taken over a single sampling duration, becoming unduly high; the output of a sampling unit is a function of $f_{\propto} \tau\left(\mathrm{I}_{1}+\mathrm{I}_{\mathrm{n}}\right)$ and so will fall or rise if the minimum sampling area or duration decreases or increases. An increase in $I_{1}$ or in $A_{1}$ might reduce $\propto$ by increasing the level of lateral inhibition; it has been shown that this falls during dark adaptation (Barlow, FitzHugh, \& Kuffler, 1957).

Although the predictions agree well with experimental spatial and temporal summation curves, they were derived for a limited set of assumptions, such as a linear transducer function and $r=+1.0$. Thus it seemed of interest to compute summation curves for different combinations of parameters, and so the program given above was modified for this purpose.

The computer was made to calculate the absolute or difference threshold for values of $t_{2}$ from 0.01 to $3.20 \mathrm{sec}$. for two values of background intensity, $I_{1}=0$ or $10^{8}$ quanta/sec. $\mathrm{deg}^{2}$, two values of $\mathrm{A}_{2}$, $0.01 \mathrm{deg} .2$ or $30 \mathrm{deg} .^{2}$, two levels of correlation, $\mathrm{r}=0$ or 0.5 , and $\mathrm{C}$ adaptation. We saw that $r$ and $\propto$. decrease as $I_{1}$ increases, not as a consequence of the present model but for reasons requiring a separate explanation. However, $\propto$ was treated as constant, being given the value $\propto=0.1 \mathrm{deg}^{2} \tau$ was made equal to 0.1 sec. for $I_{1}=0$, and 0.05 sec. for $I_{1}=10^{8}$. These values lie within the range found experimentally (Barlow, 1958). The program was modified so that, given a set of stimulus parameters, the computer first determined the corresponding number of "sampling units." The number of sensory messages generated by each sampling unit was then calculated, and the outputs were assumed to correlate and sum as before. In detail:

(1) As before it was assumed that $A_{1}=A_{2}$ or $\propto$, whichever is greater, and $t_{1}=t_{2}$ or $\tau$, whichever is greater. A sampling unit is an area $\propto$ taken over a time $\dot{r}$, and $\mathrm{N}$, the number of sampling units corresponding to a given value of $A_{1}$ and $t_{1}$, was taken as $\mathrm{A}_{1} \mathrm{t}_{1} / \propto \tau$. (For convenience, parameters which give integral values of $\mathrm{N}$ were used in the program, and it was assumed that the onset of the stimulus increment always coincided with the start of a sampling duration, and that its area always projected on to the correct number of complete sampling areas). The expected number of quantal absorptions for each summation unit was given by $x_{1}^{\prime}=f_{\alpha} r\left(I_{1}+I_{n}\right)$.

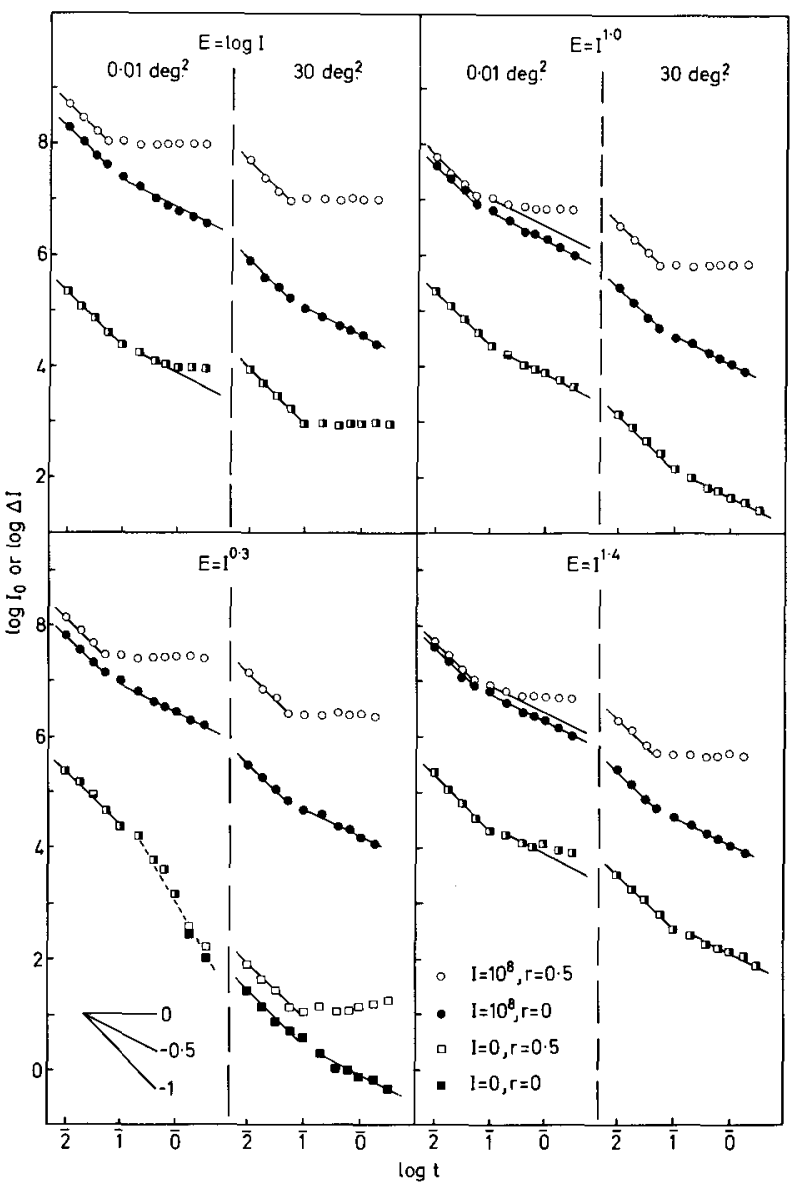

Fig. 14, $\log \Delta I\left(\log I_{0}\right.$, when $\left.I_{1}=0\right)$ is plotted against $\log t$ ( $s e c$ onds), for $E=\log I ; E=I^{0.3} ; E=I^{1.0}$, and $E=I^{1.4}$. For each transducer function curves are given for two values of $A_{2}: 0.01 \mathrm{deg}{ }^{2}$ and 30 deg. 2; two levels of correlation, $r=0$ and $r=0.5$; two values of $I_{1}$; 0 and $10^{8}$ quanta/sec.deg. 2 ; and $C$ adaptation. For $t_{2} \leq \tau$ continuous straight lines with a slope of -1.0 (Bloch's law) and for other values of $t_{2}$ lines with a slope of -0.5 (the square root law) have been fitted by eye. The dashed line fitted to one curve for $E=I^{0.3}$ and $I_{1}=0$ has a slope of -1.7 . Difference thresholds are represented by circles, and absolute thresholds by squares.

(2) For each of the $\mathrm{N}$ summation units, the actual number of quantal absorptions on the given trial, $x_{2}^{\prime}$, was taken as a variable from a Poisson distribution with mean $x_{1}^{\prime}$. ( For $x_{1}^{\prime}>100$ the normal approximation to the Poisson distribution was used.)

(3) For each of the $\mathrm{N}$ summation units, the number of absorptions which would be effective after adaptation had operated was calculated. Since $C$ adaptation was assumed, this was given by $x_{3}^{\prime}=x_{2}^{\prime}-x_{1}^{\prime}+0.05 x_{1}^{\prime}$.

(4) We now have $\mathrm{N}$ values of $\mathrm{x}_{3}^{\prime}$ which can be taken to represent the linearly summed effects of the quantal absorptions acting on each of $\mathrm{N}$ ganglion cells. The number of sensory messages generated by each ganglion cell is determined by the transducer function. The selected transducer function was applied to each of the $\mathrm{N}$ values of $\mathrm{x}_{3}{ }_{3}$ to give $\mathrm{N}$ corresponding values of $\mathrm{x}_{4}^{\prime}$, the number of nerve impulses generated by each ganglion 
cell. The four transducer functions mainly used were $x_{4}^{\prime}=\log x_{3}^{\prime}, x_{4}^{\prime}=x_{3}^{\prime 1} \cdot 4, x_{4}^{\prime}=x_{3}^{\prime}, x_{4}^{\prime}=x_{3}^{\prime 0.3}$.

The total number of sensory messages is then given by summing the outputs of the $N$ ganglion cells: $\mathbf{x}_{4}=\mathrm{N}^{\mathrm{N}} \mathrm{x}_{4}^{\mathrm{t}}$

From this point on the description of the program is identical with that given before: a value of $x_{5}$, the total central effect (E) of the stimulus on the given trial, is computed, taking into account the variance of the sensory messages, $\sigma_{\mathrm{S}}^{2}$, and the level of correlation between sensory messages. The sequence of steps to this point is repeated a 1000 times, to allow $x_{6}$, an estimate of $\mathrm{E}_{\mathrm{c}}$, the criterion which would give rise to a 2 per cent false positive rate, to be determined, and $\Delta \mathrm{I}$ or $\mathrm{I}_{\mathrm{O}}$ is calculated from this. Figure 14 shows some curves generated by this program.

This figure presents results for temporal summation for the absolute threshold, and for a difference threshold with a high background intensity, for a number of combinations of parameters. Since $t$ and $A$ behave in the same way in the equations describing the model, this figure can equally well be read as a set of predictions for spatial summation, "sec." and "deg. ${ }^{2}$ ", being interchanged wherever they occur. There is an encouraging degree of agreement between the curves for $r=0.5$ and those in Fig. 13, the effects of increase in area or background intensity, and even the order of magnitude of the thresholds, being similar. To demonstrate the effects of $r$ and $v$ on the slope of the partial summation curves for high stimulus intensities we have also calculated partial summation curves for $\tau=50$ msec., $\mathrm{I}_{1}=10^{8}$ quanta/sec. deg. ${ }^{2}, \mathrm{E}=\log \mathrm{I}, \mathrm{I}^{0.3}$, $\mathrm{I}^{0.7}$ or $\mathrm{I}^{1.0}$, and different values of $\mathrm{r}$ and $\mathrm{v}$, and the slopes between 0.1 and $3.2 \mathrm{sec}$. are given in Table $\mathrm{I}$. Since the slope of the difference threshold partial summation curve depends on the values of both $v$ and $r$, as either increases the slope gets flatter. The slopes for the uppermost curves in Fig. 13 were read from the graph as: -0.33 for $\mathrm{A}_{2}=0.011 \mathrm{deg}{ }^{2}$ and -0.03 for $A_{2}=27.6 \mathrm{deg} .^{2}$. When $v=.1$, a large value, these slopes would correspond to $r<0.1$, depending on the

Table 1. Slopes of difference threshold partial summation curves $\left(\tau=50 \mathrm{msec} . ; I_{1}=10^{8}\right.$ quanta $/$ sec. deg. $\left.{ }^{2}\right)$.

\begin{tabular}{|c|c|c|c|c|c|c|c|c|}
\hline \multirow{2}{*}{$\begin{array}{l}\text { Transducer } \\
\text { function: } \\
\mathrm{A}_{2} \text { (deg. }{ }^{2} \text { ): }\end{array}$} & \multicolumn{2}{|r|}{$\log$} & \multicolumn{2}{|c|}{0.3} & \multicolumn{2}{|r|}{0.7} & \multicolumn{2}{|r|}{1.0} \\
\hline & 0.01 & 30 & 0.01 & 30 & 0.01 & 30 & 0.01 & 30 \\
\hline$\underline{v} \quad \underline{r}$ & & & & & & & & \\
\hline $\begin{array}{ll}0.1 & 0 \\
0.1 & 0.01 \\
0.1 & 0.1 \\
0.1 & 0.2 \\
0.1 & 0.5 \\
\end{array}$ & $\begin{array}{l}-0.58 \\
-0.34 \\
-0.11 \\
-0.06 \\
-0.03\end{array}$ & $\begin{array}{c}-0.49 \\
0.01 \\
-0.02 \\
-0.03 \\
0 \\
\end{array}$ & $\begin{array}{l}-0.53 \\
-0.29 \\
-0.08 \\
-0.04 \\
-0.05\end{array}$ & $\begin{array}{l}-0.51 \\
-0.02 \\
-0.01 \\
-0.02 \\
-0.03 \\
\end{array}$ & $\begin{array}{l}-0.53 \\
-0.39 \\
-0.15 \\
-0.11\end{array}$ & $\begin{array}{l}-0.49 \\
-0.03 \\
-0.02 \\
-0.01\end{array}$ & $\begin{array}{l}-0.50 \\
-0.41 \\
-0.22 \\
-0.15 \\
-0.12 \\
\end{array}$ & $\begin{array}{l}-0.49 \\
-0.02 \\
0 \\
0 \\
0.01 \\
\end{array}$ \\
\hline $\begin{array}{ll}0.01 & 0.5 \\
0.01 & 0.8 \\
0.01 & 1.0\end{array}$ & $\begin{array}{l}-0.15 \\
-0.12 \\
-0.11\end{array}$ & $\begin{array}{c}0.01 \\
-0.02 \\
0\end{array}$ & $\begin{array}{l}-0.27 \\
-0.24 \\
-0.19\end{array}$ & $\begin{array}{l}0 \\
0.01 \\
0\end{array}$ & $\begin{array}{l}-0.47 \\
-0.43 \\
-0.35\end{array}$ & $\begin{array}{l}-0.02 \\
-0.02 \\
-0.01\end{array}$ & $\begin{array}{l}-0.49 \\
-0.43 \\
-0.45\end{array}$ & $\begin{array}{l}-0.03 \\
0 \\
-0.01\end{array}$ \\
\hline
\end{tabular}

transducer function. When $\mathrm{v}=.01$, a rather low value (Stein \& Matthews, 1965), r must be high to match the curves in Fig. 13 (for $E=I$ a larger value of $v$ would also be required).

When $r=0$ in Fig. 14, and the background intensity is high a square root law is found for all values of $t_{2}>t$ (values up to $3.2 \mathrm{sec}$, were employed). Thus, whatever the transducer function, positive correlation is necessary to explain the departure from the square root law for spatial and temporal summation beyond $\propto$ or $r$ at high background intensities. At low intensities the value of $r$ has little effect on the threshold curves except when a power transducer function with a low exponent is assumed: when $\mathrm{A}_{2}$ is also large, $\mathrm{E}=\mathrm{I}^{0.3}$ gives a higher absolute threshold curve for $r=0.5$ than for $r=0$.

When $I_{1}=10^{8}$ quanta/sec. deg. ${ }^{2}$, for all combinations of $t_{2}$ and $A_{2}$ the difference threshold increases as the exponent of the power transducer functions decreases, and it is highest for the logarithmic function, as was found previously (see Fig. 6). However, the similar slight increase which was found for the absolute threshold (see Fig. 5, C adaptation) is shown only for the same case, that is, when the increment is contained within one receptive field and sampling duration $\left(A_{2} \leq \alpha, t_{2} \leq \tau\right)$. When either the area or duration of the increment exceeds these limits, then, at these low levels of stimulation, the opposite effect is seen, $I_{0}$ falling as the exponent of the power function decreases. We saw, in an earlier discussion of transduction from the receptor to the ganglion cell, that as the number of receptors affected increases, power functions with an exponent less than one would give an enhancement effect rather than simple complete summation. A similar enhancement, produced as the number of sampling units increases, is shown here.

There are differences between the curves given by the various transducer functions in Fig. 14, and this suggests that a comparison between these curves and experimental data might give some indication of the most acceptable form for the subject's transducer function, and so provide a basis for a procedure for scaling $\mathrm{E}$. There is little to choose between the curves given by the four transducer functions when the background intensity is high, as a match to data like those in Fig. 13. The sensory noise rather than the transducer function has the predominant effect in determining their form. For each transducer function a combination of $v$ and $r$ could be found which would match the partial summation curves for the experimental data. On the other hand the absolute threshold curves are for the most part little affected by change in $r$ and show considerable differences. Thus, if we compare the "partial summation segments" (the curves for $t_{2}>r$ ) of the absolute threshold curves for $\mathrm{A}_{2}=0.01 \mathrm{deg} .^{2}$, we see that this adheres to the square root law when $E=I$, is somewhat flatter for $\mathrm{I}^{1.4}$ and $\log \mathrm{I}$, and is considerably steeper (the slope is -1.7 ) for $1^{0.3}$. On the other hand 
the absolute threshold partial summation curves for $\mathrm{A}_{2}=30$ deg. ${ }^{2}$ and $\mathrm{r}=0.5$ accord with a square root law when $E=I$, but are somewhat flatter for each of the other laws, though to varying extents. In Fig. 13 the partial summation segment of the absolute threshold curve for $A_{2}$ small and $I_{1}=0$ has a slope somewhat steeper than -0.5 , and the corresponding segment of the curve for $A_{2}$ large is flatter than the square root law would give. This suggests that the transducer function for these data is not linear, but may lie somewhere between $E=I$ and $E=I^{0.3}$, and that $E=\log I$ or power functions with exponents greater than or equal to one can be excluded.

To examine this more closely, curves for $I_{1}=0$ were computed for a number of transducer functions, and the overall slope between $\log t_{2}=\overline{1} .3$ and $\log t_{2}=$ 0.5 ( 0.2 to $3.2 \mathrm{sec}$.) was determined for both values of $A_{2}$. The slopes of the partial summation segments for Barlow's (1958) data for both spatial and temporal summation were read from his graphs. Both sets of slopes are shown in Table II. (For $A_{2}$ small the slopes for $r=0$ and $r=0.5$ were averaged together, as there were no consistent differences between them. For $\mathrm{A}_{2}$ large the slopes given are for $r=0.5$.)

The slope assigned to a partial summation curve will depend partly on the range of values of $\log t_{2}$ over which it is taken. Nevertheless, the values in Table II present a fairly clear picture. They show that for the linear transducer function the predicted slope of the temporal (ispatial) partial summation curve for $I_{0}$ is -0.5 whether $A_{2}\left(t_{2}\right)$ is small or large. For $E=\log I$, or for power functions with exponents greater than one, the partial summation curves are flatter than this for both values of $\mathrm{A}_{2}\left(\mathrm{t}_{2}\right)$. When the exponent of a power transducer function is less than one the slope of the predicted partial summation curve steepens as the exponent decreases for $A_{2}<\propto$ (or for $t_{2}<\tau$, if the table is taken to refer to spatial summation curves); but for $A_{2}>>\propto\left(t_{2}>>r\right)$ the slope becomes flatter as the exponent decreases (for $r=0.5$ ). Barlow's (1958)

Table 2. Slopes of absolute threshold partial summation curves.

\begin{tabular}{lcc}
\multicolumn{1}{c}{$\mathrm{A}_{2}:$} & 0.01 deg. $^{2}$ & 30 (or 27.6) deg. \\
\hline $\begin{array}{l}\text { Form or exponent of } \\
\text { transducer function }\end{array}$ & & \\
\hline $\log$ & & \\
1.4 & -0.22 & 0.00 \\
1.0 & -0.29 & -0.45 \\
0.9 & -0.50 & -0.52 \\
0.8 & -0.51 & -0.44 \\
0.7 & -0.59 & -0.42 \\
0.6 & -0.74 & -0.37 \\
0.5 & -0.86 & -0.20 \\
0.3 & -1.02 & -0.16 \\
\hline Barlow & -1.71 & 0.05 \\
$\quad$ (spatial) & $-\cdots .-$ & $-\cdots$ \\
Barlow & & -0.39 \\
(temporal) & -0.59 & -0.27 \\
\hline
\end{tabular}

data for temporal and spatial summation show a relation between the slopes for $A_{2}\left(t_{2}\right)$ small and $A_{2}\left(t_{2}\right)$ large which is similar to that predicted for power transducer functions with exponents less than one. His spatial partial summation slopes would suggest a transducer function with an exponent of $0.8\left(t_{2}\right.$ small) or $0.7-0.8$ ( $t_{2}$ large). His temporal summation data would suggest $0.7-0.8\left(A_{2}\right.$ small) or 0.6-0.7 (A $A_{2}$ large). Of course, these results should not be given too much weight since the effects on the predicted partial summation slopes of altering the parameters of the model require further study. Nevertheless, it is of interest that the suggested exponents lie within the range of values which has been given by a newly developed method for scaling E (Treisman, 1965), and is also within the range found for cat's optic nerve fibers by FitzHugh (1957). The comparisons made here suggest that, with further study, and provided the model presented here proves acceptable, it may be possible to use the form of partial summation curves at the absolute threshold as guides to the form of the transducer function.

Glezer (1965) has recorded spatial summation curves at the fovea during dark adaptation. He finds that as it proceeds "there is a regular increase in the zone of full summation" and partial summation increases. He concludes from this increase in partial summation that "it is evident, that the increase of spatial summation during dark-adaptation is connected with a gradual cessation of inhibition." But the results we have presented show that caution is necessary in coming to conclusions of this sort. Since the stimulus intensities used (and probably the retinal noise level) decrease during the course of dark adaptation, this alone should cause equation (7) to give place to equation (6), with a consequent increase in partial summation. Inhibition may well decrease during dark adaptation, but, if our model is accepted, Glezer's finding is not evidence that this and the level of partial summation are necessarily linked.

\section{Discussion.}

We have presented a model for visual intensity discrimination in which the central effect of a stimulus varies because of physical noise in the stimulus, the spontaneous generation of nerve impulses (which we have treated as retinal noise or "dark light") and variation in the transmission of the sensory paths (sensory noise), and this noisy central input is subject to a statistical decision procedure which determines the final response. We have shown that it is likely that the variations constituting the sensory noise are positively correlated, in contrast to the uncorrelated physical noise. At low levels of stimulation the uncorrelated noise predominates and tends to produce the square root law, but as the intensity of the background, or the area or duration of the stimulus increment increase the proportion of the total variance due to the correlated component of the sensory noise increases 
rapidly; as it becomes predominant the Weber function departs from the square root law towards a slope of 1 (in $\log -\log$ coordinates) and the spatial and temporal partial summation functions tend towards a slope of 0 . Thus the model affords a single explanation for a number of phenomena often regarded as unrelated. The basic assumptions could be applied to other modalities than vision, and should then account for most cases of Weber's law; the occurrence of this law in temporal discrimination has been explained along these lines (Treisman, 1963). To adapt the model to apply to visual intensity discrimination a number of particular assumptions were added so that precise predictions could be made which could be compared with available data. It is encouraging that the computations given above have shown that the ancillary assumptions could be considerably varied without seriously disturbing the main predictions. We have found no unavoidable consequences of the model which clearly conflict with established observations.

Certain points deserve further discussion.

(1) What is the form of the transducer function? This is equivalent to asking how to scale $\mathrm{E}$, the central effect of the stimulus. There has been much interest in the question of how "sensation" or "psychological magnitudes" can be measured or scaled (Fechner, 1860; Stevens, 1960). Fechner proposed that sensation was logarithmically related to intensity. Stevens has argued that the psychophysical law is a power function with, for brightness, an exponent of 0.3 . Stevens believes that his law describes the transducer function for the peripheral sense organs, but this appears unlikely. It has been argued that judgments about sensory magnitudes a re based on stored central representations or neural codings of stimulus magnitudes recorded on a dimension which we shall refer to as the "metric dimension," and that the so-called "direct" scaling procedures should be regarded as relating stimulus intensity to this "metric dimension." It has also been shown that the evidence that the "psychophysical law" is a power function is ambiguous, and that a better case can be made for regarding the central metric dimension as logarithmically related to stimulus intensity (Treisman, 1964c, 1964d, 1965). But in either case it follows that the form of the psychophysical law is irrelevant to the question of the proper form of the transducer function, since there is no reason to suppose that the form in which information about stimulus magnitude is coded and stored centrally is necessarily linearly related to the separate central effect of the stimulus used to select discriminative responses (Treisman, 1965). A model which appears able to explain a number of features of temporal judgment assumes that information about durations is stored in logarithmic form, but that temporal discrimination operates on a linear representation of elapsed time (Treisman, 1963).

Fechner's "sensation" is obviously analogous to E.
However, Fechner's scaling procedure was based on a model of threshold discrimination which appears inferior to the signal detection model assumed to hold here (Swets, 1964), which detracts from the weight his law can be given. There are many obscurities about "sensation;" this term has been avoided in the model presented here which gives an account of discrimination in terms which should all be ultimately identifiable with neural events. Thus if the form of the transducer function can be determined behaviorally, this information might help to guide physiological observations. The model, if correct, can helpus to identify the transducer function if it can show that some functions are compatible with experimental observations but others not.

To some extent the results we have presented above do allow us to narrow down the range of transducer functions which can be considered acceptable. The Weber functions computed were in conformity with experimental results for a number of parameter combinations provided a power transducer function was assumed. But difficulties arose with the logarithmic function. When included in the present model it produced a Weber function with an exponent greater than unity, the computed Weber fraction increasing as I increased even with zero correlation and a constant state of light adaptation (see Figs. 7 and 9). This does not altogether exclude $E=\log I$ from consideration, through it makes it appear less plausible than a power function, since it is possible to take $r=0$ or very near it and make special assumptions to make the model generate an acceptable Weber function. For example, we could require that the degree of adaptation increases as I increases at a rate just sufficient to counterbalance the rise in the Weber fraction which would otherwise occur.

The summation functions generated by the model provide information which further narrows down the range of possibilities. For all the transducer functions the results in Fig. 14 showed that if $r=0$ then the difference threshold curves at a high background intensity should follow a square root law for all values of $t_{2}>\tau$ (for temporal summation) or $A_{2}>\propto$ (spatial summation), which does not agree with the experimental data. In each case it is necessary to have $r>0$ if the partial summation difference threshold curves are to show the shift towards a zero slope which is found with experimental data. Since $r=0$ is excluded the difficulty of finding parameters that would allow the logarithmic transducer function to produce both acceptable Weber functions and difference threshold summation curves is increased, though if $v$ is large a small value of $r$ may produce considerable departure from the square root law (see Table I). However, further evidence is provided by the absolute threshold partial summation curves: when $A_{2}<\propto\left(t_{2}<\tau\right)$ the temporal (spatial) partial summation curve for $E=\log I$ would be flatter than the square root law, whereas Barlow's observa- 
tions give curves which are steeper than this. Taking all these points into consideration it appears that $\mathrm{E}=\log$ I can probably be excluded.

The absolute threshold curves in Fig. 14 allow us to go further and discriminate between the different possible power functions. If the linear law holds all partial summation curves should have slopes of $\mathbf{- 0 . 5}$, but for power functions with exponents less than one the temporal summation slope will be steeper than this for $\mathrm{A}_{2}<\alpha$, and flatter for $\mathrm{A}_{2}>>\propto$. Barlow's data followed this pattern, matching most closely the computed curves for power functions with exponents in the range $0.6-0.8$. They are clearly incompatible with the very steep slope $(-1.7)$, corresponding to marked threshold enhancement, which was given by $E=I^{0.3}$ for $A_{2}<x$. This appears to exclude power functions with exponents of this order.

The value of $0.6-0.8$ found for the exponent of the transducer function is subject to two reservations. It is an approximation which may require modification when we have better estimates of some of the parameters involved, such as $r$ (though for $A_{2}<x$ this does not affect the curves calculated). But it is unlikely that any minor change in the model would shift it as low as 0.3 , or raise it to 1.0 . Secondly, it should be regarded as applying only to Barlow's two subjects. It is quite likely that there may be considerable individual variation in the exponent of the transducer function, a problem which may repay study. However, it is of interest that it lies within the range of values which have been given by initial applications of a newly developed scaling procedure which is based on the signal detection model of the threshold (Treisman, 1965). It is a consequence of this model that the experimental relation between the Crozier ratio $(\Delta I / \sigma \Delta I$, where $\sigma \Delta I$ is the standard deviation of the threshold) and the Weber fraction depends on the form of the transducer function, and so can be used to determine the latter. Applying this procedure to Mueller's (1951) data for two subjects gave exponents of 0.5 and 0.6 , and data obtained by Blackwell and Law (1958) from four subjects gave a mean of 1.1 .

It might appear that the transducer function could be simply discovered by determining the relation of $d$ ' to $\Delta \mathrm{I}$ in experiments which allow signal detection theory to be applied (Swets, 1964). The value of $d^{\prime}$ corresponding to a stimulus $I_{2}=I_{1}+\Delta I$, where $I_{1}$ is the background or "noise" and $\mathrm{I}_{2}$ is the "signal + noise," is given by $d^{\prime}=\left(E_{2}-E_{1}\right) / \sigma_{E}$ (see Fig. 1). Tanner and Swets (1954) performed yes-no and forced-choice experiments, using intensity increments on a $10-\mathrm{ft}$. $\mathrm{L}$ background, and found that $\log d^{\prime}$ ' was linearly related to $\log \Delta \mathrm{I}$. They concluded that "change in neural activity is a power function of change in light intensity." They do not give the exponent of this power function, but it can be read from their graphs and ranges between 2.5 and 3.5, values very much greater than those we have found. However, this relation cannot be taken as the transducer function, since it depends on an arbitrary origin, $E_{1}$. If $\mathrm{E}=\mathrm{I}^{\mathrm{n}}$, and we write $\mathrm{Y}=\mathrm{E}_{2}-\mathrm{E}_{1}$ and $\mathrm{X}=\mathrm{I}_{2}-\mathrm{I}_{1}$, it does not follow that $\mathrm{Y}=\mathrm{X}^{\mathrm{n}}$ : this would require that $I_{2}^{n}-I_{1}^{n}=\left(I_{2}-I_{1}\right)^{n}$, which is only true when $n=1$. Since $Y=$ $\mathrm{g}(\mathrm{X})$ is not simply related to $\mathrm{E}=\mathrm{f}(\mathrm{I})$ we cannot readily use the former to determine the latter. Conversely, the same transducer function may give different empirical relations between $d^{\prime}$ and $\Delta I$ as $I_{1}$ varies.

It appears that if the model holds we can exclude $E=\log I$ and $E=I^{0.3}$ as possible transducer functions for visual intensity, and a power function with an exponent somewhat less than one gives the best match to the experimental data considered. This result provides us with a basis for using threshold summation curves to determine the form of the transducer function. This suggests that it would be of interest to apply both the scaling procedure based on summation curves, and the procedure which uses the relation between the Crozier ratio and the Weber fraction (Treisman, 1965) to data from the same subjects; if the two procedures converged on the same or similar functions this would greatly strengthen the arguments for their validity. However, it must be remembered that the transducer function with which we are concerned gives the relation between $\mathrm{E}$ and I for (relatively small) variations in I about a level to which the eye is adapted. (It also assumes linearity of transmission from receptor to ganglion cell, any non-linearity arising at or beyond the latter). It does not necessarily follow that the same function holds for the portion of the central effect contributed by the "adaptation level." As the results for $\mathbf{P}$ and $\mathrm{C}$ adaptation have shown, this component of $I_{1}$ can produce a logarithmic central effect or no effect and satisfactory Weber functions will still be given. The way in which light adaptation might separate this component from the effects of small stimulus fluctuations and allow a separate function to apply to each is discussed in more detail elsewhere (Treisman, 1967).

We discussed earlier the difficulties of using physiological observations as a guide to the form of the overall transducer function in a general model of the sort presented here. The problem arises because there are a number of different possible measures of the neural response to a stimulus and no obvious criterion for choosing between them. But the choice made determines the function we record: the number of impulses uccurring in a critical period may have one relation to the intensity of the stimulus evoking them, but the peak firing rate quite another. This is a problem we may be able to solve by combining behavioral and physiological observations. Thus if psychophysical procedures suggest that the transducer function has a certain form, and it is found that a particular measure of the neural response is related to stimulus intensity by the suggested function, this agreement between the functions might be taken as a reason for identifying this particular neural measure as the form in which the 
stimulus information is coded. We would then hope that if we can follow the fate in the nervous system of the nervous messages so defined, this would agree with the further assumptions of the model. It is of interest as a possible illustration of this argument that FitzHugh (1957) recorded the discharge from cats' ganglion cells to near-threshold flashes of light and found that the number of impulses occurring in a critical period following the flash was related to its intensity by a power function with exponents ranging from 0.6 to 1.2 for different cells. This agreement with the transducer function suggested by the psychophysical observations above might be taken as evidence that intensity is coded as a number of impulses. If this work is confirmed it would suggest that it might be possible to demonstrate, at some higher level in the nervous system, some process corresponding to simple summation of these impulses.

(2) The level of correlation. The assumption that there is some degree of positive correlation between the sensory messages set up by a flash of light is essential for the more important predictions given above. But it was shown that the range of possible correlations (except if very few quanta are absorbed, when, in any event, $\mathbf{r}$ does not affect the curves predicted) is effectively 0 to 1 , and the computed Weber function eventually becomes linear even if the correlation is taken as low as +0.01 . Thus any factor, however small, which would produce some degree of positive correlation would suffice to account for the form of the Weber function. A number of physiological mechanisms might have effects of this sort; a good example is the ascending reticular system. It is known that sensory inputs follow not only the classical direct paths to the appropriate areas of cortex, but also send collateral messages to the reticular formation which in turn produces more or less widespread "activation" of the cortex. This in part determines the cortical responses to the message along the primary pathways (Magoun, 1963). There is considerable convergence in the reticular formation, not only between messages in a single modality but also between signals arising in different modalities, so that the ascending effect when a stimulus is given may be considered as a sort of average of the input to the formation at that time, both from the stimulus and from other sources. Since on any trial this averaged offect will probahly produce a similar degrec of facilitation for each sensory message reaching the cortex by the direct pathways, and since the level of this activation should vary from trial to trial even if the number of sensory messages were constant (both because of noise in the reticular aderaging process, and because of variation in the components from other modalities averaged in with the effects of the flash) this should produce some degree of positive correlation between the sensory messages.

It would be of interest to know the value of $r$. At high levels of stimulation the Weber function is given by
$\Delta \mathrm{I}=\mathrm{zcvr}^{1 / 2}\left(\mathrm{I}_{1}+\mathrm{I}_{\mathrm{n}}\right)$ and the temporal partial summation curve tends to the same value (for $A_{1}=A_{2}$ ). It may be possible to estimate $c$ (see below) and $z$ can be estimated from the false positive rate, or a psychophysical procedure can be used which avoids it (Treisman, 1964a). But this still leaves both $\mathrm{v}$ and $\mathrm{r}^{1 / 2}$ to be determined from the asymptotic value of $\Delta \mathbf{I}$ in summation, or the slope of the Weber function, and it is likely that physiological evidence will be needed to separate these two parameters. Their product is the square root of the correlated portion of the variance of the sensory message, expressed as a proportion of the mean.

(3) Adaptation. Dark adaptation has not been discussed but it can also be included in the model though some difficulties remain to be resolved (Rushton, 1965a). It has been shown that at any time after exposure to a bleaching light, the threshold depends on the amount of unregenerated pigment still present; for any proportion of bleached pigment there is an "equivalent background," $I_{B}$, which gives the same threshold levels under a range of conditions (Crawford, 1947; Rushton, 1965a). It has been suggested that the bleached pigment produces effects similar to those resulting from stimulation of the eye with light (Rushton, 1963 , 1965a; Barlow, 1964); thus $\Delta I$ is high after recent exposure to a strong light because of an effective rise in $I_{n}$, the retinal noise, and the threshold fall during the course of dark-adaptation reflects the return of $I_{n}$ to its resting level as the bleached pigment regenerates. A full discussion of the retinal basis of dark and light adaptation is given elsewhere (Treisman, 1967).

A number of alternative assumptions about light adaptation, the adjustment to the background intensity, were tested in the model.

(i) Since light adaptation does occur N (no) adaptation is not a satisfactory assumption but it was included as a basis for comparison; it is clear (see Figs. 2, 3, 4, 8 and 9) that it is sufficient to allow acceptable Weber function curves to be generated in some cases.

(ii) P adaptation was included because it seemed possible that "compression" of the stimulus range to match the range of response of the nerve fiber might be achieved by transducing the main effect of $I_{1}$ logarithmically with transient departures from the "adaptation level" being transformed by some other law. The Weber functions this assumption produced were in general acceptable, falling between those for $\mathrm{N}$ and $\mathrm{C}$ adaptation (see Figs. 2, 3, 4, 11 and 12); it appeared that the law applying to departures from the "adaptation level" mainly determined their form. Levelt $(1965 \mathrm{a}, 1965 \mathrm{~b})$ found that binocular brightness averaging of suprathreshold stimuli is linear, which is not readily reconcilable with a logarithmic transformation of the mean stimulus level but does not exclude some non-linearity for ranges of stimulation other or larger than those he studied. 
(iii) In $\mathrm{C}$ adaptation the "adaptation level" produces no central effect. The deviation of the actual number of quantal absorptions on a trial from the mean number, $x_{2}-x_{1}$, plus a proportion of the mean effect, $\mathrm{cx}_{1}$ (c was taken to be 0.05 in the program), together determine the number of sensory messages generated (subject to the operation of the transducer function). No difficulties arise with this assumption; it appears the most satisfactory of those made, though many alternatives could be proposed. To the extent that this description of light adaptation is satisfactory it suggests a number of advantages that the process may have for the organism: (a) We saw, in Figs. 11 and 12, that the main effect of $\mathrm{C}$ adaptation is to slow down the transition from the square root to the linear law, thus giving lower thresholds at the higher intensities. Information about the magnitude of the background stimulation is preserved if the corresponding message is always scaled down to $\mathrm{cx}_{1}$, but the amount of sensory noise generated at the next stage is reduced. This reduction lowers the threshold and a higher level of background stimulation is necessary before the cor related sensory noise can become predominant and make the Weber function linear. (b) Light adaptation and other factors such as the presence of receptors with different ranges of operation (the rods and cones) allow the large range of light intensities which the eye can discriminate to be matched to the response capabilities of ganglion cells. Thus a transducer function such as the logarithmic, which could also do this but which would entail low differential sensitivity at high intensities, can be avoided. (c) It may keep the firing rate of ganglion cells near the optimal level at which they respond best to increments or decrements. At half the maximum firing rate the signals (impulse or space) are equiprobable and so their mean information is highest, but the optimal rate is most probably that at which $\mathrm{v}$ is minimal. When the fiber is firing at a high rate, and difficult to excite further, or is held down to a very low rate, it is possible that $\mathrm{v}$ may increase.

It seems likely that the mechanisms producing the initial phase of light adaptation are closely related to or identical with those which reduce $\propto$ and $t$ as $\mathrm{g}\left(\mathrm{I}_{1}+\mathrm{I}_{\mathrm{n}}\right)$ increases. Treisman (1964a) and Glezer (1965) have suggested that this reduction is due to feedback. It may serve to prevent the ganglion cell firing rate (which is a function of $f_{\times} \times\left(I_{1}+I_{n}\right)$ ) rising too high, which, we have suggested, is one function of the processes underlying light adaptation. It is probable that $\propto$ is reduced by an increase in lateral inhibition, and this, as well as other mechanisms (Treisman, 1967), may also contribute to the fall in $\mathrm{c}$ from $\mathrm{c}=1$ (the vilue before adaptation) to the resting level, $c_{O}$, in adurtation

It high levels of stimulation the Weber fraction may start to increase, and this increase may be prevented by surrounding the photometric comparison field with a large field of a similar order of luminance (Steinhardt, 1936; Holway, 1937; Pirenne, 1962a). Possibly at these high values of $\mathrm{I}_{1}$ inhibitory activity in the area illuminated reaches a maximum. Then if $I_{1}$ increases further the rise in excitation is no longer countered by a corresponding increase in inhibition, so that $c$ rises (see equation (4)), which would increase the slope of the Weber function. If adaptation declines the rate of firing of the ganglion cells may rise, which might increase $\mathrm{v}$ and reduce the response to increments, an effect equivalent to a fall in the exponent of the power transducer function. Both these changes would also contribute to the disproportionate rise in $\Delta I$. The addition of the illuminated surround may prevent this departure from Weber's law by recruiting further lateral inhibition and thus returning $c$ to its previous value.

It would be of interest to estimate c. Baker (1949) followed the course of light adaptation during $15 \mathrm{~min}$. exposure to an adapting light in subjects who had been previously dark-adapted. He found that $\Delta \mathrm{I}$ dropped to a minimum in about $3 \mathrm{~min}$. , and then rose slightly to a resting level which it reached in 10 or $15 \mathrm{~min}$. Assuming equation (4), we can attribute these changes to a decline in $\mathrm{c}$ from a value of 1 at the light-onset when there is effectively no adaptation to the final resting level, $c_{0}$, and the difference between the threshold at light onset and the final resting level can be used to give $c$, an estimate of $c_{0}$. High adapting intensities, for which equation (4) applies, should all, if the model is correct, give the same value for 'ć, since $c_{o}$ is constant. At very low light intensities equation (2) applies and $c_{0}$ does not enter into the determination of the threshold, so we should get $\hat{\mathrm{c}}=1$. Intermediate values of $I_{1}$, at which the transition from equation (2) to equation (4) occurs, should give intermediate values of c. Baker used four adapting intensities, 5, 50, 500 and 5000 trolands, and the corresponding mean values of c for his two subjects, as given by the difference between the initial threshold and the final level after $15 \mathrm{~min}$. adaptation, were: $0.75,0.44,0.48,0.47$. These results show the initial high value and a fall to a more or less constant level which we expected, but the actual values are very high. However, they are certainly overestimates: Baker's initial threshold reading was in each case an average of measures taken over the first quarter minute. During this time the threshold is falling very rapidly, so that his average must be considerably less than the true initial threshold which would correspond to $c=1$. Crawford (1947) found that the threshold fell about $1.5 \mathrm{log}$ units in the first $0.4 \mathrm{sec}$. of exposure to a $100 \mathrm{c} / \mathrm{ft}^{2}{ }^{2}$ conditioning stimulus. Further experiment is clearly needed to provide a basis for accurate assessment of $c$, but Baker's observations suggest that the relation to $I_{1}$ is in general agreement with expectation.

(iv) In $M$ adaptation $c=0$ so that only deviations from the mean effect of $I_{1}$ produce sensory messages. 
Consequently there is no transition from equation (2) to (4) and the former applies to all values of $I_{1}$. This should produce lower thresholds at the high intensities than any other assumption, but it would also require a second retinal system which would respond to absolute levels of intensity. Since the square root law does not apply for all values of $I_{1}$, the assumption that $\mathrm{c}=0$ must be rejected.

(v) In Fractional (F) adaptation it was assumed that the number of quantal absorptions on each trial was reduced to a constant proportion: $\mathrm{x}_{3}=\mathrm{kx}_{2}, 0<\mathrm{k}<1$. As an account of light adaptation this is unsatisfactory since it implies that adaptation is as rapid a process as excitation, so that the reduction in response at any moment is directly proportional to the actual input at that time, whereas we know that light adaptation is a relatively slow process. However, the relation $\mathrm{x}_{3}=\mathrm{kx}_{2}$ could serve as a description of the reduction in the number of quantal absorptions effective in producing sensory messages that would occur if the quantum efficiency of the bleaching of visual pigment by light were less than one, and it is of interest for this reason. This is discussed below.

(4) Quantum efficiency. We have implicitly assumed that each quantum absorbed bleaches the corresponding molecule of visual pigment, i.e., that the quantum efficiency, $\gamma$, defined as the ratio of the number of chromophores dissociated to the number of quanta absorbed, is one, but there is some evidence against this (Dartnall, 1957). Hagins (1955) illuminated the rabbit's retina with flashes less than about $1 \mathrm{msec}$. in duration and found that no matter how intense the flash was, it bleached no more than half the rhodopsin. This seemed to imply a maximum quantum efficiency no greater than 0.5 , although the result is consistent with much smaller values. According to Williams (1965) as the flash intensity increases the quantum efficiency is reduced because of the occurrence of photo-reversal of bleaching: if a molecule absorbs an odd number of quanta it bleaches, but if it absorbs an even number within a sufficiently short period it does not bleach. Thus $\gamma$ depends on the intensity of the light: at low intensities $\gamma$ is one, but as intensity increases $\gamma$ tends to zero and the proportion of pigment bleached by a short flash tends to 0.5 .

The effectiveness of light in stimulating the eye does not decline at the same rate as the quantum efficiency of bleaching. As the intensity of light increases quanta will be absorbed but will fail to bleach the molecule because their effects are immediately cancelled by absorption of a second quantum. But the receptor output which signals the absorption of a quantum of light does not depend on the bleaching of the molecule which has absorbed the quantum, but more probably arises from the isomerization of the chromophore or during the conversion of pre-lumirhodopsin to metarhodopsin (or their equivalents for other visual pigments). Thus on some occasions a molecule of visual pigment may absorb a quantum of light, emit a light signal, and then be reisomerized to the original form by absorption of a second quantum; the two quanta absorbed would not contribute to bleaching (and so would reduce $\gamma$ ) but they would contribute to signalling the absorption of light. Thus if we define the "quantum efficiency of light signal generation," $\delta$, as the ratio of the number of light signals produced to the number of quanta absorbed, this will fall when multiple absorptions occur but should not fall as much as $\gamma$. (However, it must be borne in mind that some authors have discussed $y$ as though it were an index of light signal generation).

What effect would $\delta$ have on the threshold? This would be represented, in the model, by $x_{3}=\delta x_{2}, 0<\delta<1$, where $x_{3}$ now stands for the number of light signals emitted, and $x_{2}$ is the number of quanta absorbed, on a given trial. The relation $x_{3}=0.005 x_{2}$, which has been presented as "Fadaptation" exactly corresponds to this, and the $F$ curves in Figs. 11 and 12 can be considered as representing the effects of a constant value of $\delta=0.005$. As the $\mathrm{N}$ curves, for which $\delta=1$, and the $\mathbf{F}$ curves were identical, it follows that the decrease in $\delta$ as $I_{1}$ increases has no effect on the threshold.

This absence of effect on intensity discrimination is surprising. It occurs because the effect of introducing $\delta$ into the model as we have done is merely to rescale $\mathrm{x}_{2}$. The mean number of light signals generated becomes $\delta \mathrm{g}_{1}\left(\mathrm{I}_{1}+\mathrm{I}_{\mathrm{n}}\right)$ and its variance is $\delta^{2} \mathrm{~g}_{1}\left(\mathrm{I}_{1}+\mathrm{I}_{\mathrm{n}}\right)$. This makes equation (1)

$$
\Delta I=\frac{z}{\delta g} \sqrt{\delta 2_{g} 2 v 2\left(\left.\right|_{1}+I_{n}\right)^{2}+\delta{ }^{2} g\left(l_{1}+I_{n}\right)+\delta 2 \sqrt{2} g\left(I_{1}+l_{n}\right)}
$$

which simplifies to equations (2) and (3) exactly as before, $\delta$ disappearing. However, this argument is based on treating $\delta$ as a constant, whereas the proportions of molecules absorbing different numbers of quanta will obviously vary, even when the total number of quanta absorbed is constant. We can neglect the slight correlation that must exist between $\delta$ and the fluctuations in the number of quanta absorbed from a constant stimulus and allow for this variation by treating $\delta$ in the same way as $f$, i.e., we can take the number of light signals generated to be a Poisson variable with mean and variance equal to $\delta \mathrm{g}\left(\mathrm{I}_{1}+\mathrm{I}_{n}\right.$ ) (Aguilar \& Stiles, 1954). When we do this equation (2) becomes

$$
\Delta I=\frac{z}{\delta^{1 / 2} g^{1 / 2}}\left(I_{I^{+}} I_{n}\right)^{1 / 2}
$$

but equation (3) remains unchanged. (Strictly $\delta$ will be slightly less for $I_{2}$ than for $I_{1}$ so that equation (3) should really be increased by $\delta_{1} / \delta_{2}$, but this fäctor would be only negligibly greater than one and can be ignored). But when the fraction of rhodopsin bleached by $I_{1}$ is small $\delta$ (and even more so, $\delta^{1 / 2}$ ) will be very close to one. Even if each quantum absorbed bleached one molecule, a retinal illumination one million times threshold (50 trolands) would bleach only one per cent of the rhodopsin in an hour (Pirenne, 1962b) so that 
the quantum efficiency of bleaching would be negligibly less than one (Williams, 1965), yet at this level of illumination the Weber function would be linear. Thus the conclusion suggested by the $F$ curves remains unchanged. At low levels of stimulation $\delta$ appears in equation (2) but is little less than one so that its effect on the threshold is negligible. As $\mathrm{I}_{1}$ increased $\delta$ decreases, but it also disappears from the equation describing the Weber function, and thus does not affect the difference threshold.

Of recent years the work of Rushton (1965a) and others has obliged us to discard the notion that the rise in threshold subsequent to exposure to a bleaching light is wholely attributable to a reduction in the amount of pigment available to catch quanta. If the present arguments are accepted, we can drop altogether the hypothesis that changes in the efficiency with which quanta are absorbed from the incident light, and used to generate light signals, play a part in raising the dark adaptation threshold. By elimination, this further favors the hypothesis that bleached pigment raises the threshold in the dark by increasing the retinal noise. Immediately after exposure to a strong light the proportion of unbleached pigment will be low so that there will be a reduction in the number of quanta absorbed from a given light stimulus. The effect of this on the production of light signals can be regarded as exactly equivalent to the effect which would be produced if the pigment complement were maintained unchanged but $\delta$ fell. But since the noise level will be high equation (3) will apply (with $I_{B}$ substituting for $\left(I_{1}+I_{n}\right)$ ) and $\delta$ does not appear in this. When most of the pigment has regenerated equation (2) will come to apply, but now the reduction in $\delta$ which would have an effect equivalent to the loss of pigment would be small and $\delta^{1 / 2}$ would be very close to one. (Of course if the pigment level were to fall and no retinal noise were created, so that levels of stimulation could remain relatively low, the threshold would rise, to an extent depending on the value of $\delta$ equivalent in its effect to the pigment fall.)

"Quantum efficiency"' is sometimes used to refer to the efficiency of the eye as a whole (really the subject) in performing some task, as compared with the performance of an ideal device limited only by the quantum variability of light. Barlow (1962a) defines the overall quantum efficiency of the eye as

$\mathrm{F}=$ Least quantity of light theoretically required for performing a task

Least quantity required in practice for performing that 'same task

The tasks usually used are the detection of an increment (Jones, 1959) or discrimination between two stimuli differing in intensity. Barlow (1962b) employed the latter task. It is possible to calculate the minimum number of quanta that each stimulus must provide if an ideal device limited only by the Poisson variability of light is to discriminate between them at the same level of performance as the subject. The ratio of this value to the actual mean number of quanta delivered to the subject's eye (the ratio is the same for each stimulus) is then the overall quantum efficiency, $F$ 。 Barlow found a maximum value of about 5 per cent for this.

It is obvious that $\mathbf{F}$ is a complex measure, affected by all restrictions on performance other than that resulting from the Poisson variability of light. A major restriction is the loss of light occurring between arrival at the cornea and absorption by the rods; in the model this is represented by the constant, f. Rushton (1956) estimated that about 10 per cent of the light incident at the cornea was absorbed by visual pigment. If this restriction were excluded from consideration, the quantum efficiency would be given by $F / f$, which would make Barlow's maximum about 50 per cent. Barlow considers the retinal noise, $I_{n}$, and $\gamma$, the quantum efficiency of bleaching, as the remaining restrictions on performance. However, we have seen that $\gamma$ can be disregarded. Furthermore, there seems no good reason to suppose that visual discriminations are wholly determined at the retina, or that the brain beyond the retina is completely noiseless. In terms of the model presented here the two sources of biological noise, retinal and sensory, should together account for $\mathrm{F} / \mathrm{f}$ falling short of 1 . If $\sigma_{\mathrm{s}}^{2}$ and $\mathrm{I}_{\mathrm{n}}$ were both zero $\mathrm{F} / \mathrm{f}$ would equal one, but the bigger the proportionate contribution of these sources of noise to $\sigma \frac{2}{E}$, the smaller will the overall quantum efficiency be. A number of consequences follow from the model which it is of interest to compare with Barlow's (1962b) results. (a) If $r$ is positive the proportionate contribution of the sensory noise to the central variance increases as $g_{1}\left(I_{1}+I_{n}\right)$ increases and this should cause $F$ to fall. Barlow's findings are in keeping with this: $F$ is maximum near the absolute threshold and it falls if the intensities of the two stimuli to be discriminated are made higher, even when the eye is adapted to their order of intensity. (b) Light adaptation allows $\mathrm{c}$ to fall to a resting level, $\mathrm{c}_{o}$. This reduction in the contribution of the adapting intensity to the magnitude of the central message reduces the amount and proportion of correlated central noise which contributes to $\sigma_{\mathrm{E}}^{2}$. It follows that $\mathrm{F}$ should be greater when the eye is adapted to the intensity of the stimuli to be discriminated than when it is not. Barlow showed that this is so. (c) We have suggested that there may be an optimal firing rate for the optic nerve fiber al which $\mathbf{v}$ is minimal, and that one function of light adaptation may be to maintain the firing rate of the ganglion cell for stimuli in the threshold range near this rate. In accordance with this Barlow found that as the intensity of the two stimuli was shifted above or below the intensity threshold for the adapting field, $F$ also varied, being highest near threshold and falling off at the intensities on either side of this level. Thus Barlow's three main results using this measure are all in agreement with the model. 


\section{Conclusion}

The model that has been presented was constructed in the belief that any adequate account of sensory discrimination must make minimum assumptions about every stage between the impingement of the stimulus on the receptors and the choice of the final response, and that we must attempt to make the assumptions precise at all stages. It is dangerous to assume that because more is known about the retina than about the brain beyond it, the latter can be assumed to have no effect on discriminatory performance and safely ignored; or that references to "conscious sensation" allow us tacitly to assume linearity and noiselessness beyond the periphery. We have also tried throughout to maintain a distinction between explanations and descriptions, important when using terms such as "adaptation" or "summation." The model is somewhat schematic: it was desired to test its general usefulness as a framework to which further assumptions could be added if particular problems required them. Many of the assumptions employed have already given useful service elsewhere; the number used seemed the minimum which would allow the construction of an adequate model. Thus no attempt was made to take account of the shift from rod to cone vision, though this could readily be included by assuming an appropriate change in parameters. We have not considered the effects of overlap between receptive fields though it is likely that this occurs and is important; it might be related to some of the differences that may be found between spatial and temporal summation. We have dealt only with incremental thresholds determined by the method of constant stimuli, but our arguments could be extended without difficulty to the other psychophysical procedures for measuring thresholds (Treisman, 1964a).

The model appears to be able to explain a number of features of discrimination and leads to some results, such as the irrelevance of $\delta$ to the increment or dark adaptation threshold, which might not otherwise have been suspected. It demonstrates that observed changes in a complex system do not necessarily imply that corresponding changes are occurring in underlying causal factors which have a simple one-to-one relation to the observed phenomena. An underlying "summation process" does not become less efficient as the parameters of the increment increase: noise is sampled as before but its description changes. The lesser degree of light adaptation shown when the adapting light is weak does not imply that $c$ is larger than at high intensities: the finding is sufficiently explained by the shift from equation (4) to equation (2) which reduces the weight of $\mathrm{c}$ in determining the threshold. The increase in partial spatial summation that occurs during the course of dark adaptation (Glezer, 1965) does not need to be explained by a corresponding decrease in lateral inhibition; it would follow from the shift from equation $(3)$ to equation $(2)$ as the retinal output $\left(\mathrm{I}_{\mathrm{B}}\right)$ falls, even if lateral inhibition did not decrease.

The model may prove useful in suggesting predictions and experiments. Thus it will be of interest to attempt to estimate all the constants in the model. Procedures can be suggested for estimating $z, c, f$, and $I_{n}$ (Treisman, 1964a; Rushton, 1956; Gregory, 1956; Barlow, 1957), and it may be possible to obtain separate estimates of $v$ and $r$ by combining behavioral and physiological observations. In determining the transducer function it may be possible to compare two separate scaling procedures, one based on the Weber function (Treisman, 1965), the other on the absolute threshold partial summation curve, and examine whether they converge on the same result. As well as allowing individual variation to be studied, determination of the transducer function by behavioral means may facilitate the interpretation of physiological observations, as was discussed above.

It may prove possible to derive results from the model which otherwise might have seemed to require special assumptions. A possible case in point is the observation that "at high intensities the Weber fraction $\Delta \mathrm{I} / \mathrm{I}$ of the cone system as a whole ... becomes constant (Weber's law) and has about the same value for light of all spectral compositions" (Pirenne, 1962a). A possible explanation for this is that there are a number of cone systems (Stiles, 1959) and that at high intensities the incremental threshold is determined by the most sensitive of them. However, let us consider what the present model would predict. We will suppose that the wave-length of $\Delta \mathrm{I}, \lambda_{2}$, may differ from that of $\mathrm{I}_{1}, \lambda_{1}$. Since the capture of quanta of light by the receptors is a function of wave-length it follows that we will have different values of $f$ in each case. Thus to include variation in wavelength among the determinants of the Weber function at high intensities we can generalize equation (4) to

$$
\Delta I=\frac{f_{1} A_{1}{ }^{\dagger} 1}{f_{2} A_{2}{ }^{\dagger}} \quad \operatorname{ver}^{1 / 2}\left(I_{1}+f_{n}\right)
$$

(If $\mathrm{f}_{1}$ changes than $\mathrm{I}_{n}$, the "dark light" corresponding to the rate of spontaneous generation of impulses in the visual system, should change too. But at high intensities this can be ignored.) It follows that for $A_{1}=A_{2} \geq \propto$ the resulting Weber function is independent of $A_{2}$, for $t_{1}=t_{2} \geq \tau$ it is independent of $t_{2}$, and for $f_{1}=f_{2}$ the Weber function is the same whatever the actual spectral composition of the light, provided the level of stimulation is high. A prediction which follows from equation (8) is that when $f_{1}<f_{2} \Delta I$ will be lower, and when $f_{1}>f_{2}$ it will be higher than when $f_{1}=f_{2}$, and the effect on the threshold is given by $f_{1} / f_{2}$. It follows that interchanging the wave-lengths used as background and increment may have a marked effect on $\Delta \mathbf{I}$. The hypothesis that the single most sensitive mechanism determines all thresholds when the level of stimulation is high is quite compatible with the present model, 
but we see that if the model is accepted the observation quoted above does not require this hypothesis to explain it but follows simply from equation (8).

\section{Summary}

A model for visual intensity discrimination is described. The main assumptions are: (i) The absorption of quanta from a light-flash by the retinal receptors is subject to fluctuations due to the physical variability of light; (ii) absorbed quanta may give rise to neural messages; (iii) retinal noise also gives rise to neural messages; (iv) the number of neural messages depends on the "overall transducer function" relating the central nervous effect (E) of the stimulus to its physical intensity (I): $E=f(I)$, and on (v) the state of light adaptation; (vi) sensory noise affects the magnitude of the neural messages; (vii) the magnitudes of the sensory messages generated by a given light-flash are positively correlated; (viii) the sensory messages sum to give the final central effect, $\mathrm{E}$, and the response selected is determined by a statistical decision procedure.

Many of these assumptions are already accepted or are plausible. To examine their predictions when taken together, and the effects of variation in the parameters and functions assumed, the model was simulated on a digital computer. It appears that it correctly predicts the relation between the difference threshold, $\Delta I$ and the intensity of the background stimulus, I (the Weber function), found experimentally, and it also predicts a number of the features of retinal summation, including the effects of increase in background intensity, and stimulus area and duration, on partial temporal or spatial summation. Evidence is provided that the overall transducer function is not a logarithmic function or a power function with a small exponent, and a new basis for scaling the sensory effect of a stimulus is suggested. It is shown that Weber's law arises if there is any degree of positive correlation between sensory messages, but not if there is zero correlation, and possible mechanisms of light adaptation are considered.

The assumptions which allow Weber's law to be derived for vision are sufficiently general to be capable of being applied to other sensory systems.

\section{References}

Aguilar, M., \& Stiles, W. S. Saturation of the rod mechanism of the retina at high levels of stimulation. Optica Acta, 1954, $1,59-65$.

Alpern, M. Rod-cone independence in the after-flash effect. $J$. Physiol. 1965, 176, 462-472.

Baker, H. D. The course of foveal light adaptation measured by the threshold intensity increment. J. Opt. Soc. Amer., 1949, 39 , $172-179$.

Barlow, H. B. Summation and inhibition in the Frog's retina. $J$. Physiol., 1953, 119, 69-88.

Barlow, H. B. Retinal noise and absolute threshold. J. Opt. Soc. Amer., 1956, 46, 634-649.

Barlow, H. B. Increment thresholds at low intensities considered as signal/noise discriminations. J. Physiol., 1957, 136, 469488

Barlow, H. B. Temporal and spatial summation in human vision at different background intensities. J. Physiol., 1958, 141 337-350.

Barlow, H. B. A method of determining the overall quantum efficiency of visual discriminations. J. Physiol., 1962a, 160, 155168

Barlow, H. B. Measurements of the quantum efficiency of discrimination in human scotopic vision. J. Physiol., 1962b, 160, 169 188.

Barlow, H. B. Dark-adaptation: a new hypothesis. Vision Res. $1964,4,47-58$.

Barlow, H. B., Fitzhugh, R., \& Kuffler, S. W. Change of organization in the receptive fields of the cat's retina during dark adaptation. J. Physiol., 1957, 137, 338-354.

Berelson, B., \& Steiner, G. A. Human behavior. An inventory of scientific findings. New York: Harcourt, Brace \& World; 1964.

Birdsall, T. G. The theory of signal detectability. In H. Quastler (Ed.), Information theory in psychology. Goencoe, Ill.: Free Press, 1955.

Blackwell, H. R., \& Law, O. T. A study of possible "photosensitization" "f the human eye by white light. Engng. Rsch. Inst. Rep. No. 2455-9-F. University of Michigan, June, 1958.

Brindley, G. S. Physiology of the retina and the visual pathway. London: Edward Arnold, 1960.

Cattell, J. M. On errors of observation. Amer. J. Psychol., 1893, $5,285-293$.

Crawford, B. H. Visual adaptation in relation to brief conditioning stimuli. Proc. Roy. Soc., 1947, 134B, 283-302.

Crouzy, M. Sur la limite absolue imposée par les fluctuations quantiques au seuil differentiel de luminance. Comptes rendus des Séances de L'Académie des Sciences, 1961, 253, 559-561.

Dartnall, H. J. A. The visual pigments. London: Methuen, 1957.

Fechner, G. T. Elemente der Psychophysik. Leipzig: Breitkopf \& Hartel, 1860 .

FitzHugh, R. The statistical detection of threshold signals in the retina. J. gen. Physiol, 1957, 40, 925-948.

Glezer, V. D. The receptive fields of the retina. Vision Res., $1965,5,497-525$.

Gregory, R. L. An experimental treatment of vision as an information source and noise channel. In E. C. Cherry (Ed.), Information theory. Third London Symposium. London: Butterworth, 1956.

Hagins, W. A. The quantum efficiency of bleaching of rhodopsin in situ. J. Physiol., 1955, 129, 22-23P.

Hartline, $\mathbf{H}$. K. Intensity and duration in the excitation of single photoreceptor units. J. cell. comp. Physiol., 1934; 5, 229-247.

Hartline, H. K. The effects of spatial summation in the retina on the excitation of the fibers of the optic nerve. Amer. J. Physiol. $1940,130,700-711$

Hecht, S., Shlaer, S., \& Pirenne, M. H. Energy, quanta and vision. J. gen. Physiol., 1942, 25, 819-840.

Holway, A. H. On the precision of photometric observations. $J$. Opt. Soc. Amer., 1937, 27, 120-123.

Jones, R. C. Quantum efficiency of human vision. J. Opt. Soc Amer., 1959, 49, 645-653.

Keller, $\mathbf{M}$. The relation between the critical duration and intensity in brightness discrimination. J. exp. Psychol., 1941, 28, 407418 .

Le Grand, Y. Light, colour and vision. London: Chapman \& Hall, 1957.

Levelt, W. J. M. Binocuiar brightness averaging and contour information. Brit. J. Psychol., 1965a, 56, 1-13.

Levelt, W. J. M. On binocular rivalry. Institute for Perception RVOTNO, Soesterberg, The Netherlands: $1965 \mathrm{~b}$.

Magoun, H. W. The waking brain. (2nd ed.), Springfield, Ill.: Thomas, 1963.

Matin, L. Binocular summation at the absolute threshold of peripheral vision. J. Opt. Soc. Amer., 1962, 52, 1276-1286.

Mueller, C. G. Frequency of seeing functions for intensity discrimination at various levels of adapting intensity. $J$ : gen. Physiol., $1951,34,463-474$.

Neyman, J., \& Pearson, E. S. On the problem of the most efficient tests of statistical hypotheses. Phil. Trans. Roy. Soc. A, 1933, 
$231,289-337$.

Piéron, H. Vision in Intermittent Light. In W. D. Neff (Ed.), Contributions to sensory physiology. Vol. I. New York: Academic Press, 1965. Pp. 179-264.

Pirenne, M. H. Physiological mechanisms of vision and the quantum nature of light. Biol. Rev., 1956, 31, 194-241.

Pirenne, M. H. Liminal brightness increments. In H. Davson (Ed.), The eye, Vol. 2. The visual process. New York: Academic Press, $1962 \mathrm{a}$.

Pirenne, M. H. Dark-adaptation and night vision. In H. Davson (Ed.), The eye. Vol. 2. The visual process. New York: Academic Press, $1962 b$.

Ratliff, F. Mach bands: Quantitative studies on neural networks in the retina. San Francisco: Holden-Day, 1965.

Rose, $\boldsymbol{A}$. The sensitivity performance of the human eye on an absolute scale. J. Opt. Soc. Amer., 1948, 38, 196-208.

Rushton, W. A. H. The rhodopsin density in the human rods. $J$. Physiol., 1956, 134, 30-46.

Rushton, W. A. H. Peripheral coding in the nervous system. In W. A. Rosenblith (Ed.), Sensory communication. New York: Wiley, 1961. Pp. 169-181.

Rushton, W. A. H. Increment threshold and dark adaptation. J. Opt. soc. Amer., 1963, 53, 104-109.

Rushton, W. A. H. Visual Adaptation. Proc. Roy. Soc. B. 1965a, $162,20-46$.

Rushton, W. A. H. The sensitivity of rods under illumination. $J$. Physiol., 1965b, 178, 141-160.

Shallice, $\mathbf{T}$. The detection of change and the perceptual moment hypothesis. Brit. J. statist. Psychol., 1964, 17, 113-35.

Solomons, L. M. A new explanation of Weber's law. Psychol. Rev. $1900,7,234-240$.

Stein, R. B., \& Matthews, P. B. C. Differences in variability of discharge frequency between primary and secondary muscle spindle afferent endings of the cat. Nature, 1965, 208, 1217-1218.

Steinhardt, J. Intensity discrimination in the human eye. I. The relation of $\Delta \mathrm{I} / \mathrm{I}$ to intensity. J. gen. Physiol., 1936, 20, 185-209.

Stevens, S. S. On the psychophysical law. Psychol. Rev., 1957, $64,153-181$

Stevens, S. S. The psychophysics of sensory function. Amer. Scientist., 1960, 48, 226-253.

Stiles, W. S. Color vision: the approach through increment-threshold sensitivity. Proc. Nat. Acad. Sci., 1959, 45, 100-114.

Stroud, J. M. The fine structure of psychological time. In H. Quastler (Ed.), Information theory in psychology. Glencoe, Ill: The Free Press, 1955.

Swets, J.A. (Ed.), Signal detection and recognition by human observers. New York: Wiley, 1964.

Swets, J. A., Tanner, W. P., \& Birdsall, T. G. Decision processes in perception. Psychol. Rev., 1961, 68, 301-340.

Tanner, W. P., \& Swets, J. A. A decision-making theory of visual detection. Psychol. Rev., 1954, 61, 401-409.

Thurstone, L. L. Psychophysical analysis. Amer. J. Psychol., $1927,38,368-389$.

Treisman, $M$. Temporal discrimination and the indifference interval: implications for a model of the "internal clock." Psychol. Monogr. , 1963, 77, (Whole No. 576).

Treisman, M. Noise and Weber's Law: the discrimination of brightness and other dimensions. Psychol. Rev., 1964a, 71, 314-330.

Treisman, M. The effect of one stimulus on the threshold for another: an application of signal detectability theory. Brit. J. statist. Psychol., 1964b, 17, 15-35.

Treisman, M. Sensory scaling and the psychophysical law. Quart. J. exp. Psychol., 1964c, 16, 11-22.

Treisman, M. What do sensory scales measure? Quart. J. exp. Psychol, 1964d, 16, 387-391.

Treisman, M. Signal detection theory and Crozier's law: derivation of a new sensory scaling procedure. J. Math. Psychol., 1965, $2,205-218$.

Treisman, M. Dark adaptation, light adaptation and retinal noise. 1967, (in preparation).
Treisman, M., \& Watts, T. R. The relation between signal detectability theory and the traditional procedures for measuring sensory thresholds: estimating d' from results given by the method of constant stimuli. Psychol. Bull., 1966 (in press).

White, C. T. Temporal numerosity and the psychological unit of duration. Psychol. Monogr., 1963, 77, (Whole No. 575).

Williams, T. P. Rhodopsin bleaching: Relative effectiveness of high and low intensity flashes. Vision Res., 1965, 5, 633-638.

Wolf, E., \& Zigler, M. J. Course of dark adaptation under various conditions of pre-exposure and testing. J. Opt. Soc. Amer., 1955, 45, 696-702.

\section{Note}

1. I would like to thank Professor R. C. Oldfield for provision of research facilities, the Medical Research Council for their support and the Director of the Computing Laboratory for facilities afforded. I would particularly like to thank Mrs. Joyce Clarke for her invaluable assistance with programming and $I$ would also like to express my appreciation to A. W. Ingleton for useful conversations.

\section{Glossary of Symbols}

« : the limiting area below which complete spatial summation occurs; the minimum sampling area.

$\mathrm{A}_{1}$ : area of the background stimulus in deg.

$\mathrm{A}_{2}$ : area of the stimulus increment in deg. ${ }^{2}$

$C$ adaptation : Complete adaptation; $x^{3}=x_{2}-k x_{1}=x_{2}-x_{1}+c x_{1}$

$c$ : the proportion of the mean number of molecular decompositions effective in setting up sensory messages after $C$ adaptation (for $\mathrm{N}$ adaptation $c=1$; for $\mathrm{M}$ adaptation, $c=0$ ).

$\Delta \mathrm{I}:$ the difference threshold, the brightness increment detected on 50 per cent. of trials.

$E$ : the sum of the sensory messages arriving centrally on a given trial; the decision axis for the determination of the response. Corresponds to $x_{5}$ in the programme.

$E_{1}$ : the mean value of $E$ for stimulus $I_{1}$

$E_{2}$ : the mean value of $E$ for stimulus $I_{2}$

$\mathrm{E}_{\mathrm{c}}$ : the criterion determining the response.

$F$ : overall quantum efficiency of the eye. The ratio of the least quantity of light theoretically required for performing a task to the least quantity required in practice.

$F$ adaptation : Fractional adaptation, $x_{3}=h x_{2}$.

$f:$ the proportion of the quanta incident at the cornea which is absorbed by visual pigment.

$f_{1}$ : the value of $f$ for the background intensity, $I_{1}$

$\mathrm{f}_{2}$ : the value of $f$ for the stimulus increment, $\Delta \mathrm{I}$

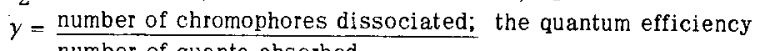
number of quanta absorbed

$\mathrm{g}=\mathrm{fAt}$

$\mathrm{g}_{1}=\mathrm{fA} \mathrm{A}_{1}$

$g_{2}=\mathrm{fA}_{2} \mathrm{t}_{2}$

$\mathrm{h}$ : the proportion of the molecular decompositions on a given trial effective in setting up sensory messages. See $F$ adaptation

$I_{1}$ : the intensity of the background stimulation in quanta/sec.deg. ${ }^{2}$.

$I_{2}$ : the intensity of the increment plus background.

$I_{B}$ : the equivalent background having the same effect as the retinal noise after exposure to a bleaching light.

$\mathrm{I}_{\mathrm{n}}$ : the "dark light", the intensity which would produce quantal absorptions at the same rate as the retinal noise produces events confusable with them.

$k$ : the proportion of the mean number of molecular decompositions constituting the 'adaptation level' in $\mathrm{P}$ and $\mathrm{C}$ adaptation.

$\lambda=$ the wavelength of light

$\lambda_{1}=$ the wavelength of $I_{1}$

$\lambda_{2}=$ the wavelength of $\Delta I$

$M$ adaptation : Adaptation to the mean; $x_{3}=x_{2}-x_{1}$.

$\mathrm{N}$ : The number of summation units contained in a given sampling area and duration.

$\mathrm{N}$ adaptation: No adaptation; $\mathrm{x}_{3}=\mathrm{x}_{2}$

$\mathrm{O}_{\mathrm{r}}$ : the output of a single receptor cell

$\mathrm{P}$ adaptation: Partial adaptation; $\mathrm{x}_{3}^{\prime}=\mathrm{x}_{2}-\mathrm{kx} \mathrm{x}_{1}$, and $\mathrm{x}_{3}^{\prime \prime}=\mathrm{kx} \mathrm{x}_{1}$.

$\mathrm{P}(\mathrm{Y})$ : the probability of the response 'Yes' on trials on which a stimulus is presented. 
$P\left(Y / I_{1}\right)$ : the value of $P(Y)$ for trials on which stimulus $I_{1}$ is presented.

$Q$ : the total number of quanta absorbed from a given stimulus. $Q_{n}$; the total number of quanta absorbed to reach threshold when light from the stimulus is distributed over $n$ receptors.

$r$ : the mean level of correlation between the sensory messages arising at a given time.

$\sigma_{\mathrm{E}}^{2}$ : the variance of the central effects produced by repeated presentations of a given stimulus.

$\sigma_{\mathrm{s}}^{2}:$ the variance of a sensory message

$s$ : the mean number of nerve impulses constituting a sensory message; $s=1$ in the programme

$\tau:$ the limiting duration below which complete temporal summation occurs.

$t_{1}$ : the duration of the background stimulus in sec.

$t_{2}$ : the duration of the stimulus increment in sec.

$\mathrm{v}$ : the coefficient of variation of the sensory message; $v=\sigma s / \mathrm{s}$ $\mathrm{x}_{1}$ : the expected number of molecular breakdowns when a flash is given including quantal absorptions and the effects of the "dark light": $x_{1}=g_{1}\left(I_{1}+I_{n}\right)$.

$\mathrm{x}_{2}$ : the actual number of molecular breakdowns on a given trial; a variable from a Poisson distribution with mean $=x_{1}$.

$\mathrm{x}_{3}$ : the number of molecular breakdowns contributing to determining the neural message, after light adaptation has operated.

$\mathrm{x}_{4}$ : the number of sensory neural messages set up by a given stimulus input.

$\mathrm{x}_{5}$ : the total number of nerve impulses arriving centrally on a given trial; this corresponds to $\mathrm{E}$.

$x_{6}$ : the value exceeded by 2 per cent. of the computed values of $\mathrm{x}_{5}$ for a given set of conditions; an estimate of $E_{c}$

$z$ : a standardized normal deviate.

(Received in the Editorial Office May 9, 1966.) 